\title{
Impact of GPS Radio Occultation Refractivity Soundings on a Simulation of Typhoon Bilis (2006) upon Landfall
}

\author{
Mien-Tze Kueh ${ }^{1}$, Ching-Yuang Huang ${ }^{1,{ }^{*}}$, Shu-Ya Chen ${ }^{1}$, Shu-Hua Chen ${ }^{1,2}$, and Chien-Ju Wang ${ }^{1}$ \\ ${ }^{1}$ Department of Atmospheric Sciences, National Central University, Chung-Li, Taiwan, ROC \\ ${ }^{2}$ Department of Land, Air and Water Resources, University of California, Davis, California, USA
}

Received 27 June 2007, accepted 21 January 2008

\begin{abstract}
Typhoon Bilis which struck Taiwan in July 2006 was chosen to assess the potential impact of GPS radio occultation (RO) refractivity soundings on numerical simulation using the WRF model. We found that this case elucidates the impact of the limited GPS RO soundings on typhoon prediction due to their favorable locations. In addition, on top of available precipitable water (PW) and near-surface wind speed from SSM/I data, we have also explored their combined impacts on model prediction.

The two GPS RO soundings available from FORMOSAT-3 near the model initial time are assimilated using the nonlocal operator in WRF 3DVAR. More contributions after the assimilation are found in the moisture increments (up to $-1.2 \mathrm{~g} \mathrm{~kg}^{-1}$ ) surrounding the corresponding occultation positions to the east of the typhoon vortex and southwest of the subtropical high, with dryness occurring in the lower troposphere. The assimilation of PW induces much larger and wider moisture increments to the west of the typhoon center. The ingestion of near-surface wind speeds produces more pronounced temperature and wind increments at lower levels.

The SSM/I data improve the predictions of typhoon intensity and intense rainfall over Taiwan, especially for the later simulation time, but generally have a less impact on track prediction as compared to the GPS RO data. Assimilation of the two GPS RO soundings leads to a marked improvement on the track that otherwise will be southward biased prior to landfall and also enhances the positive impact when the SSM/I data have been assimilated as well. An improvement using the GPS RO data is also found for daily rainfall but only for larger thresholds at later times when the bias in track in the control experiment is remedied. In general, the combined assimilation of both GPS RO and SSM/I data has shown the most promising performances. Finally, the sensitivity tests indicate that the closer GPS RO sounding to the east of the typhoon plays a more crucial role in the positive impact on track prediction.
\end{abstract}

Key words: FORMOSAT-3, GPS radio occultation refractivity, Data assimilation

Citation: Kueh, M. T., C. Y. Huang, S. Y. Chen, S. H. Chen, and C. J. Wang, 2009: Impact of GPS radio occultation refractivity soundings on a simulation of Typhoon Bilis (2006) upon landfall. Terr. Atmos. Ocean. Sci., 20, 115-131, doi: 10.3319/TAO.2008.01.21.03(F3C)

\section{INTRODUCTION}

The assimilation of satellite data would be helpful for improving numerical weather prediction since satellite data provide observations over data-poor regions such as open oceans. Through radio occultation (RO) with linkage to many low-earth-orbiting satellites (LEOs), the Global Positioning System (GPS) satellites take such an advantage to provide valuable information about the state of the atmosphere across the globe. Various forms of variables can be retrieved from the GPS RO observations, ranging from raw

\footnotetext{
* Corresponding author

E-mail:hcy@atm.ncu.edu.tw
}

excess phases, bending angle, and refractivity to retrieved moisture and temperature profiles. The accuracy of the GPS RO data retrieval has been examined by a number of studies (e.g., Rocken et al. 1997, 2000; Kuo et al. 2004, 2005), and is generally compatible with that of conventional radiosonde soundings.

Studies with GPS RO data assimilation of both bending angle and refractivity have shown a positive impact on regional as well as global weather predictions (e.g., Kuo et al. 1997, 2000; Zou et al. 1999, 2000; Liu and Zou 2003). Recently, Huang et al. (2005) have shown that both track and accumulated rainfall predictions can be improved in simula- 
tion of two typhoons impinging Taiwan when GPS RO refractivity soundings have been assimilated.

By the assimilation of GPS RO refractivity, vertical profiles of atmospheric moisture and temperature can be well modulated to improve the model initial conditions and ensuing prediction. On the other hand, assimilation of precipitable water (PW) from Special Sensor Microwave/ Imager $(\mathrm{SSM} / \mathrm{I})$, which is the integrated water vapor in the vertical column, can also improve rainfall predictions (e.g., Kuo et al. 1996; Chen et al. 2004). Chen et al. (2004) assessed the impact of SSM/I data on hurricane simulations and showed that the simulated storm's intensity was greatly improved due to the modification of the moisture content in the atmosphere and the increase of the low-level cyclonic circulation. Both GPS and SSM/I provide valuable observations over the ocean, and both are complementary to each other. The SSM/I data can retrieve the total amount of the integrated moisture (i.e., PW) but not a vertical profile which can be retrieved at a resolution of about $200 \mathrm{~m}$ from GPS RO measurements. However, due to the very limited number of the current LEOs, the GPS RO measurements provide the global observations at a much coarser resolution (e.g., about 300 - $500 \mathrm{~km}$ for COSMIC) in comparison with a swath of measurements at horizontal resolution of only several tens of $\mathrm{km}$ from SSM/I. The impacts of assimilation of these unconventional data with such different spatial resolutions on severe weather prediction are worthy of investigation. In addition, due to the fact that the GPS RO data can not provide wind information, it is also meaningful to explore the impact of the SSM/I-retrieved near-surface wind speed on severe weather prediction.

In earlier studies, a local operator has been used to assimilate the observed Abel-retrieved refractivity, which is assumed to represent a local model value at a perigee point (e.g., Kuo et al. 1997; Huang et al. 2005). Such a local operator may result in larger errors over regions where significant horizontal moisture or temperature gradients exist as discussed in Sokolovskiy et al. (2005a). To reduce the errors of representativeness, they suggest use of the integrated amount of refractivity along the ray path, namely the excess phase $(E P H)$, as the new observable for assimilation. This new operator assimilating excess phase is the so-called nonlocal operator since it takes into account the nonlocal effect of horizontal gradients along the entire path within the model depth (Sokolovskiy et al. 2005b). As found, the nonlocal operator produces considerably smaller fractional errors than the local operator using the point refractivity. The new nonlocal operator has been developed and implemented into the WRF-VAR (version 2.1) for the Weather Research and Forecasting (WRF) Model by Chen et al. (2009).

The launch of six FORMOSAT-3/COSMIC (FORMOSA Satellite \#3/Constellation Observing System for Meteorology, Ionosphere and Climate) satellites on 15 April 2006 provides more available GPS data for regional model assimilation. In this study, we adopted the new nonlocal operator in WRF-VAR assimilation system to assess the impact of GPS RO refractivity from FORMOSAT-3/COSMIC satellites on Typhoon Bilis in July 2006. The case of Typhoon Bilis illustrates a great impact of one valuable GPS sounding near the typhoon vortex on typhoon track and rainfall predictions, as will be presented later in this paper. The main goal of this study will not aim at the comparisons for the two operators and detailed analyses of the behaviors of the nonlocal operator (for these please see our companion paper of Chen et al. 2009). Rather, we would like to show convincing results to the community that assimilation of GPS RO soundings may give a marked improvement on typhoon prediction. Hence, we will focus on the impacts of GPS RO soundings on simulated typhoon's track and intensity, and the associated rainfall in Taiwan. These impacts by GPS RO soundings will be further explored in addition to the SSM/I data (both PW and near-surface wind speed). To clarify the impact of each RO sounding, sensitivity tests on removal of a particular sounding are also conducted. Section 2 gives an introduction of Typhoon Bilis (2006), the ingested data, and the experimental design in this study. The assimilation and model forecast results are presented in section 3. Finally, our conclusions are given in section 4 .

\section{EXPERIMENT DESIGN}

\subsection{Typhoon Bilis}

Typhoon Bilis (2006) originated from a tropical depression that formed on 8 July to the west of Guan. The depression strengthened into a typhoon two days later as it moved northwestward and was named Bilis. Typhoon Bilis moved northwestward consistently and made landfall in northeast Taiwan on 13 July. After crossing straight over northern Taiwan, Bilis struck the southeastern China later on $14 \mathrm{July}$, and then weakened into a tropical depression inland the next day. During 13 to 15 July, Bilis brought torrential rainfalls to Taiwan. A daily rainfall maximum up to $680 \mathrm{~mm}$ in south Taiwan was recorded by the CWB (the Central Weather Bureau) in Taiwan.

\subsection{Model Settings and Experiment Design}

To simulate Typhoon Bilis, we adopted the WRF model (version 2.1.2) with three nested domains at 45-, 15-, and $5-\mathrm{km}$ resolutions, respectively (Fig. 1a). The model has 31 vertically-stretched sigma levels with a model top at $50 \mathrm{hPa}$. The detailed descriptions of the WRF model are given by Skamarock et al. (2005). The model simulations are conducted with the Kain-Fritsch's cumulus parameterization scheme (Kain 2004) in the two outer domains, and the explicit cloud scheme of Purdue Lin (Chen and Sun 2002) in all the three domains. The YSU scheme is applied for PBL parameterization (Hong et al. 2006). No vortex "bogussing" 
or relocation is applied in our simulations. The effect of a bogus vortex on typhoon prediction has been investigated in Chen et al. (2009). We intend herein to purify the sole impact by realistic observations.

Typhoon Bilis (2006) occurred in the earlier adjustment stage of the six FORMOSAT-3/COSMIC satellites after launch. Before Bilis made landfall in Taiwan, only two GPS $\mathrm{RO}$ events were available to the east of the Typhoon Bilis at 0141 UTC and 0218 UTC 12 July, respectively. Hence, the model was initialized at 0000 UTC 12 July within $3 \mathrm{~h}$ of which the two GPS RO soundings in the outermost model domain become legible for assimilation. The best track of Typhoon Bilis (from the CWB) during 0000 UTC 12 to 0000 UTC 15 July is shown in Fig 1a. The locations of the two GPS RO soundings are marked by crosses (Fig. 1). The RO location nearer to the initial typhoon center is located at $138.0^{\circ} \mathrm{E}$ (called sounding 1 ) with the occultation time at 0218 UTC, while the other one is located at $154.8^{\circ} \mathrm{E}$ (called sounding 2), just southwest of the subtropical high.

Alternately, there is a swath of SSM/I data (shaded) passing over the marginal seas of the East Asia in a twohour assimilation window centered at 0000 UTC 12 July (Fig. 1a). Unfortunately, there is no coverage on the typhoon vortex for the SSM/I observations near the initial time. The $\mathrm{SSM} / \mathrm{I}$ data are assimilated to explore the benefit of additional wind information, as well as the huge amount of data density in moisture fields, compared with the GPS RO soundings. The retrieved PW $\left(\mathrm{g} \mathrm{cm}^{-2}\right)$ and oceanic near- surface wind speed are assimilated in this study. Both products from SSM/I are available only over the ocean and to the west of the typhoon vortex, and are retrieved from the brightness temperatures using Wentz's SSM/I Benchmark Pathfinder Algorithm at the Global Hydrology Resource Center (GHRC). These products are obtained from the measurements of the SSM/I instruments aboard the Defense Meteorological Satellite Program (DMSP) F-13, F-14, and F-15 satellites. The SSM/I low frequency channels V22, V37, and H37 are used in the retrieval algorithm, where suffixes $\mathrm{V}$ and $\mathrm{H}$ represent the vertical and horizontal polarizations, respectively. Detailed information of the SSM/I instrument and Wentz's SSM/I algorithm can be found in Hollinger (1989) and Wentz (1993), respectively.

The new nonlocal operator, which has been implemented into WRF-VAR (version 2.1) by Chen et al. (2009), is adopted to assimilate the GPS refractivity in this study. The descriptions of the WRF 3DVAR can be found in Barker et al. (2003, 2004). The methodology of the nonlocal operator as well as the statistical estimation of the observation errors are given in detail by Chen et al. (2009). Information about SSM/I data assimilation, including the observational operator and error variances, can be found in Chen et al. (2004).

The initial first guess for WRF-VAR is taken from the National Centers for Environmental Prediction (NCEP) Aviation Model (AVN) global analysis, which is also used for the boundary conditions in all the experiments in this
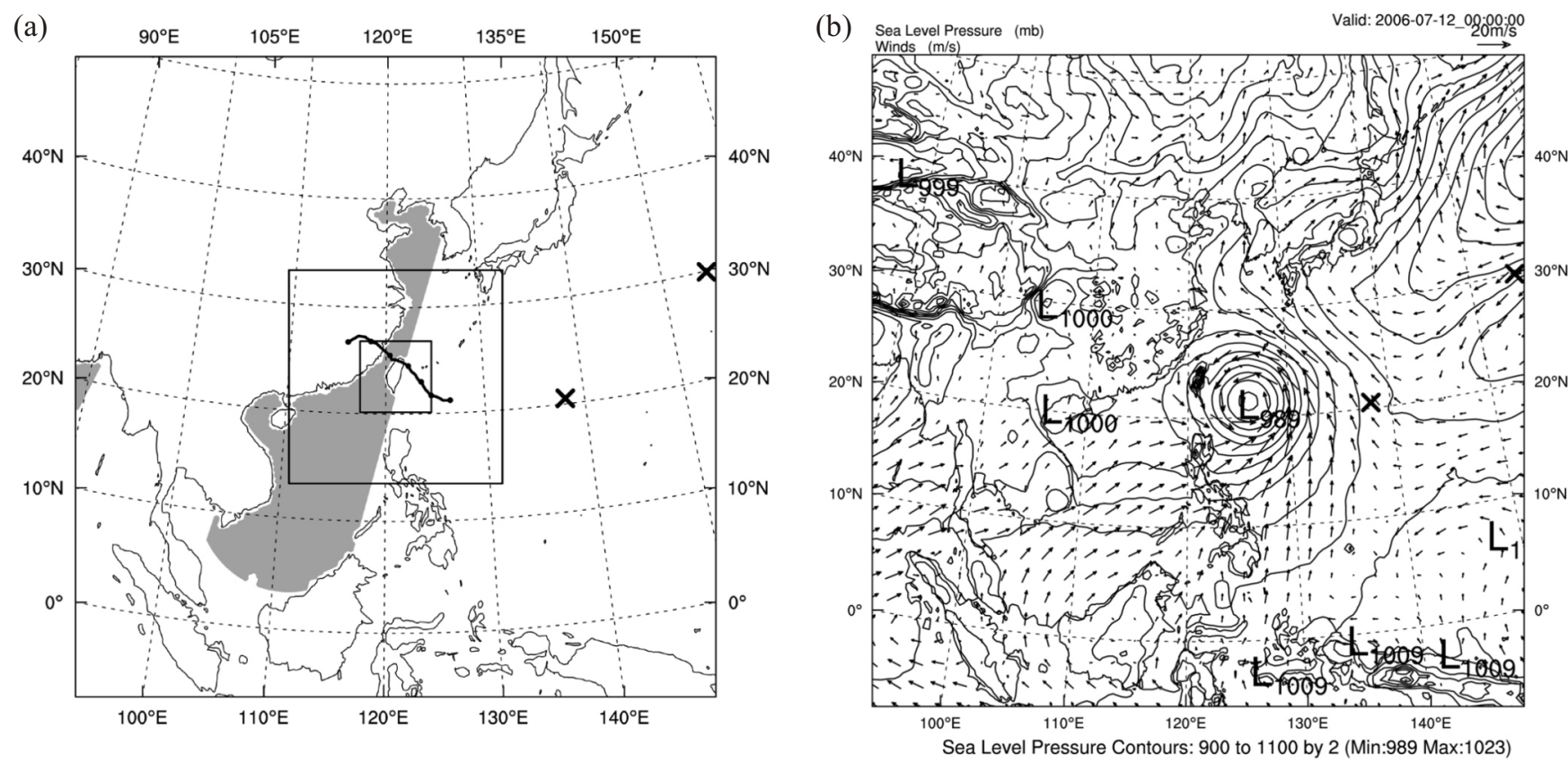

Fig. 1. (a) Three model simulation domains with horizontal resolution of 45,15 , and $5 \mathrm{~km}$, respectively. The best track from the CWB during the simulation period (dotted-line), and the coverage of SSM/I data swaths (shaded area) are superimposed. (b) Sea level pressure (hPa) and near surface wind $\left(\mathrm{m} \mathrm{s}^{-1}\right)$ in the outermost domain at the initial time (0000 UTC 12 July 2006). The contour interval is $2 \mathrm{hPa}$. The reference wind vector is indicated over the top of the panel. The locations of the two GPS soundings are marked by crosses. 
study. The near-surface wind and the sea-level pressure from the NCEP AVN analysis at 0000 UTC 12 July 2006 are shown in Fig. 1b. The central low pressure of $989 \mathrm{hPa}$ for Typhoon Bilis is slightly weaker than the observation $(984 \mathrm{hPa})$ at this time as inferred by the CWB. The two GPS RO soundings (marked by crosses in Fig. 1b) are located within the southwest periphery of the subtropical high, where the steering flow of the typhoon may prevail. Furthermore, the SSM/I data swath only covers the western part of the outer circulation of Typhoon Bilis and the southwesterlies over the South China Sea. Numerical experiments were designed to study the impacts of the GPS RO soundings and SSM/I data on Bilis' simulations.

Table 1 gives the summary of our numerical experiments conducted in this study. The simulation without assimilating any data (denoted by NONE) is our control experiment. The assimilation of GPS RO soundings using the nonlocal operator in EPH is performed in the outermost domain since these soundings are present only in this domain. The name "SSMI" denotes the experiment assimilating both PW and wind speed data, while "SSMIpw" indicates the experiment assimilating PW data only. The assimilation of the SSM/I data is performed for each domain. To discuss the impact of assimilation of individual GPS RO sounding, two sensitivity experiments (EPHr1 and EPHr2) are conducted, in which the GPS RO soundings 1 and 2 are removed, respectively. The integration time is $72 \mathrm{~h}$ for all the experiments.

\subsection{Analyses of Initial Increments}

Since GPS RO refractivity in the neutral atmosphere is primarily determined by both temperature and moisture, the initial increments of these two variables produced by the assimilation of GPS RO refractivity soundings will be analyzed herein. The initial increments produced by SSM/I data assimilation are also presented for comparisons. The initial increments are defined as the differences between the initial fields with and without the specific data assimilation.
To examine whether the profiles of initial fields fit more to the GPS RO observation after assimilation, the fractional differences in excess phase between the analysis (A, analysis after assimilation), background (B, first guess), and the observation $(\mathrm{O})$ are presented in Figs. 2a and $\mathrm{b}$ for soundings 1 and 2, respectively. The lowest height (near the surface) for sounding 1 is much lower than sounding 2 . The height of original observations may be up to $50 \mathrm{~km}$, but herein the results (in fractional change) can be shown only below the model top. For both soundings, the fractional differences of $\mathrm{O}-\mathrm{B}$ decrease with height below $5 \mathrm{~km}$, with the maximums amount exceed $1 \%$ at their bottom levels. The departures of the initial first guesses from the observations have been reduced significantly after assimilation, as seen for the profile of O-A (dotted-dashed). This improvement is more pronounced at lower levels where refractivity is much larger. The large deviations of O-A at upper levels (above $10 \mathrm{~km}$ ) and lower levels (below $5 \mathrm{~km}$ ) clearly indicate more inaccuracy of the AVN analysis.

According to the statistical observation errors from our companion paper (Fig. 1b in Chen et al. 2009), the refractivity from the Abel inversion (local operator) has much larger fractional errors at lower levels. However, the errors are much reduced for the excess phase (assimilated by the nonlocal operator) for which the refractivity is integrated along a straight line as a new observable. Currently, the operators at the European Centre for Medium-Range Weather Forecasts (ECMWF) and NCEP are local operators which may use either the RO observations as local refractivity or local bending angle, and thus limit the usage of the RO data below $5 \mathrm{~km}$ or so (Healy and Thépaut 2006; Cucurull et al. 2007). However, there are many studies (e.g., Ware et al. 1996 for GPS/MET; Kuo et al. 2005 for CHAMP) indicating that the level of the accuracy of RO data is compatible to that of conventional soundings. Therefore, larger fractional errors existing in the RO refractivity by no means indicate that the data should be completely discarded. But, it does indicate that the use of these data must be more careful by taking

Table 1. Descriptions of the numerical experiments.

\begin{tabular}{lll}
\hline Experiments & \multicolumn{1}{c}{ Assimilated data } & Assimilated domains \\
\hline NONE & None & None \\
EPH & GPS refractivity & Outermost domain \\
SSMI & PW and WS from SSM/I & All domains \\
EPH+SSMI & GPS refractivity, PW and WS from SSM/I & All domains \\
EPH+SSMIpw & GPS refractivity, PW from SSM/I & All domains \\
\hline Sensitivity Experiments & & \\
\hline EPHr1 & GPS refractivity with the sounding at $138.0^{\circ}$ E removed & Outermost domain \\
EPHr2 & GPS refractivity with the sounding at $154.8^{\circ}$ E removed & Outermost domain \\
\hline
\end{tabular}


larger observation errors into account for assimilation of such information into the model. In this study, we have rather limited observations (only two RO soundings) and we have used the nonlocal operator to simulate the new observable. To account for the larger fractional errors at lower levels as indicated in Chen et al. (2009), smaller weighting coefficients at lower heights have been used. A data quality control is also implemented in WRF 3DVAR, and any data will be rejected when the data exceed a certain threshold (five times the O-B in this case). This implementation is to allow use of the data within the error range depending on the value of O-B. Furthermore, the fractional errors of excess phase in Chen et al. (2009, their Fig. 1) are reasonably small (less than 3\%) in a statistical sense. The deviation of $\mathrm{O}$ from
B is less than 2\% for both soundings as seen in Fig. 2 in this study, thus indicating that both the observations, with reasonably specified errors, can still be used to modulate the background field.

For absolute changes due to assimilation, larger moisture increments are found below $5 \mathrm{~km}$ for both soundings (Figs. 2c, d), with their peak values at about 2.5 and $3 \mathrm{~km}$, respectively. Most of these increments produced by the GPS RO soundings are not greatly influenced by other data (SSMI and SSMIpw) assimilations. This is due to the fact that both the GPS RO and SSM/I data are not collocated in the same region. It is also interesting to point out that, at the location of sounding 1 , the peak value of the initial moisture increments for $\mathrm{EPH}+\mathrm{SSMI}$ is somewhat larger than those for (a)

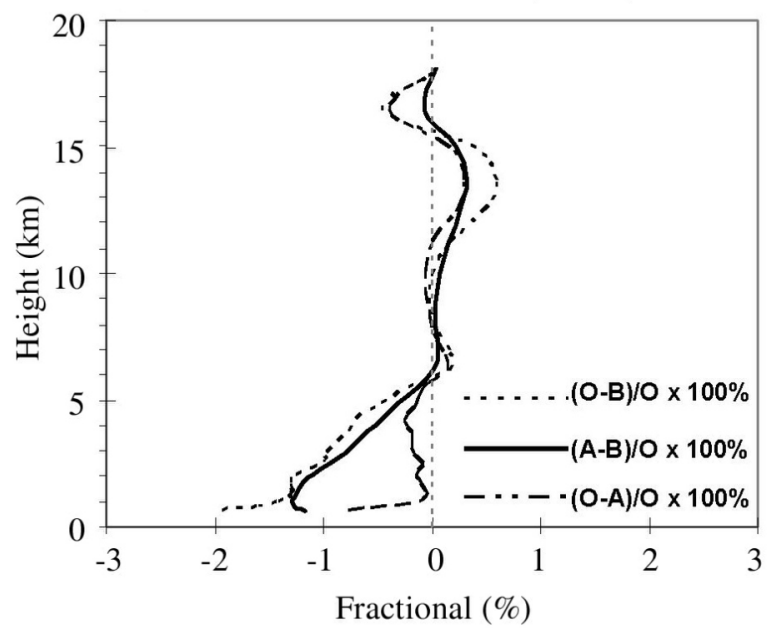

(c)

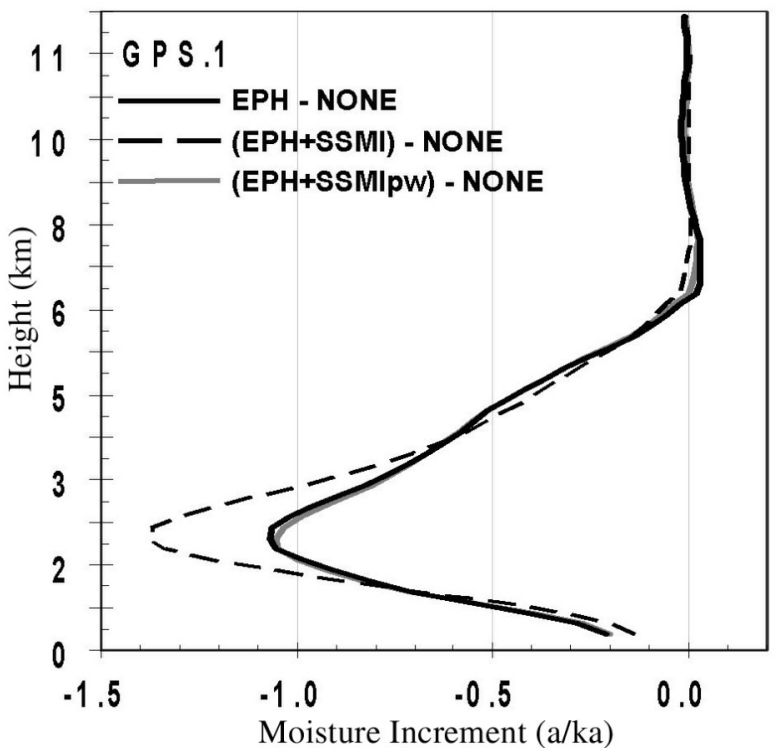

(b)

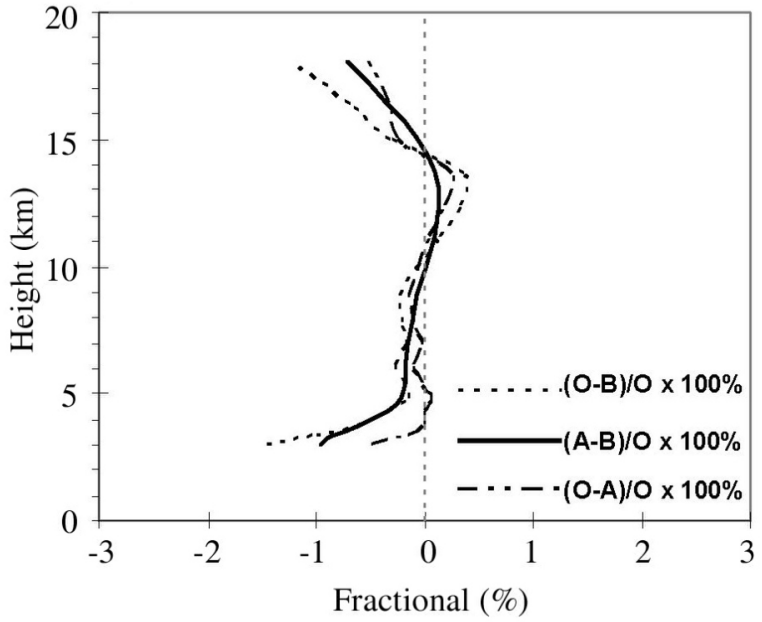

(d)

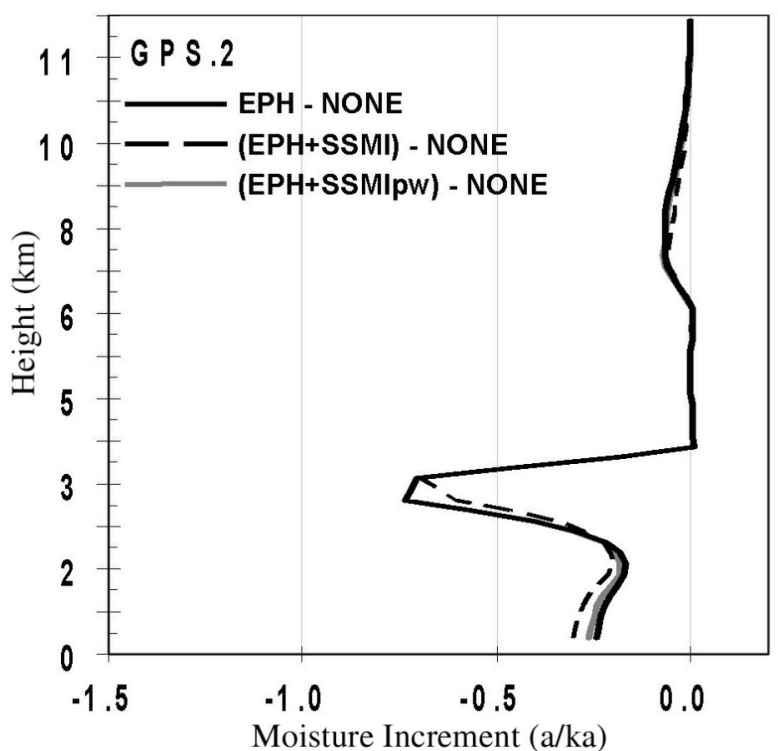

Fig. 2. The fractional difference of the excess phase by the nonlocal operator for the GPS RO sounding 1 (a) and sounding 2 (b). The fractional values are $(\mathrm{O}-\mathrm{B}) / \mathrm{O} \times 100 \%$ (dotted line), $(\mathrm{A}-\mathrm{B}) / \mathrm{O} \times 100 \%$ (solid line), and $(\mathrm{O}-\mathrm{A}) / \mathrm{O} \times 100 \%$ (dotted-dashed line), where $\mathrm{A}, \mathrm{B}$, and $\mathrm{O}$ represent the analysis, background, and observation fields, respectively. (c) and (d) as in (a) and (b), respectively, but for the initial increments of water vapor ( $\mathrm{g} \mathrm{kg}^{-1}$ ) for experiments EPH (solid line), EPH+SSMI (dashed line), and EPH+SSMIpw (grey solid line). 
EPH and EPH+SSMIpw. For sounding 1 at a location less away from the SSM/I impact region, the combined assimilation (with wind speed data) may result in additional changes in moisture increment.
In additional to the vertical profiles, the horizontal distributions of the initial increments are also examined. The initial increments for EPH at $\sigma=0.83$ (about $838 \mathrm{hPa}$ ) are shown in Fig. 3a. The two soundings produce positive tem- (a) perturbation potential temperature $(\mathrm{K})$ at Eta Level=10
Water vapor mixing ratio $(\mathrm{g} \mathrm{kg}-1) \quad$ at Eta Level $=10$

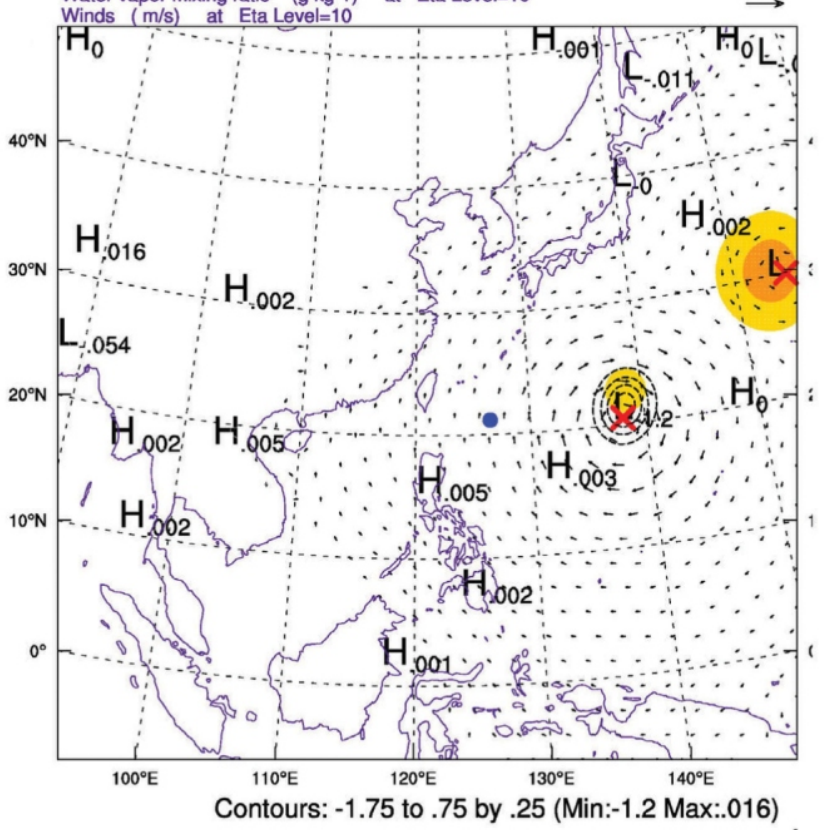

(c) perturbation potential temperature $(\mathrm{K})$ at Eta Level=10
Water vapor mixing ratio $(\mathrm{g} \mathrm{kg}-1)$ at Eta Level=10

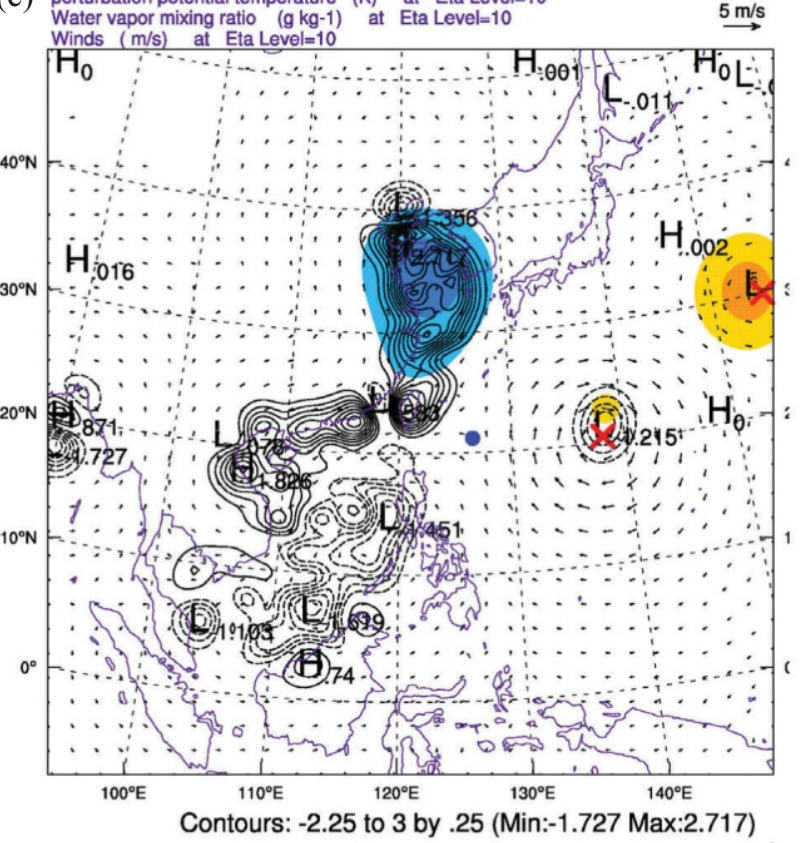

(b) perturbation potential temperature (K) at Eta Level $=10$

Water vapor mixing ratio $(\mathrm{g} \mathrm{kg}-1)$ at Eta Level=10 $5 \mathrm{~m} / \mathrm{s}$

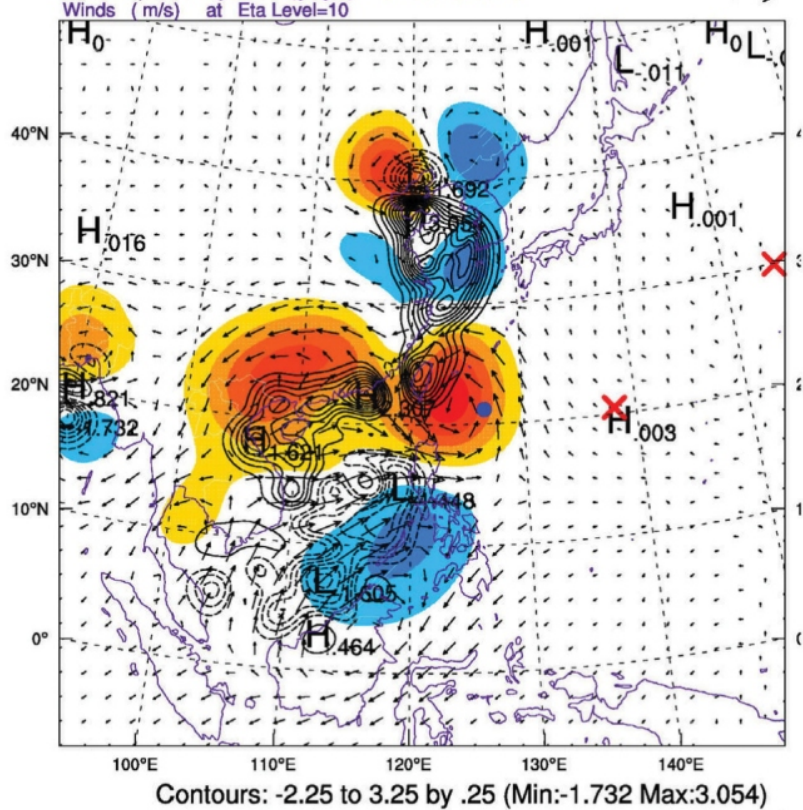

(d) perturbation potential temperature (K) at Eta Level=10 Water vapor mixing ratio $(\mathrm{g} \mathrm{kg}-1)$ at Eta Level $=10$ Winds (m/s) at Eta Level=10 $\stackrel{5 \mathrm{~m} / \mathrm{s}}{\longrightarrow}$

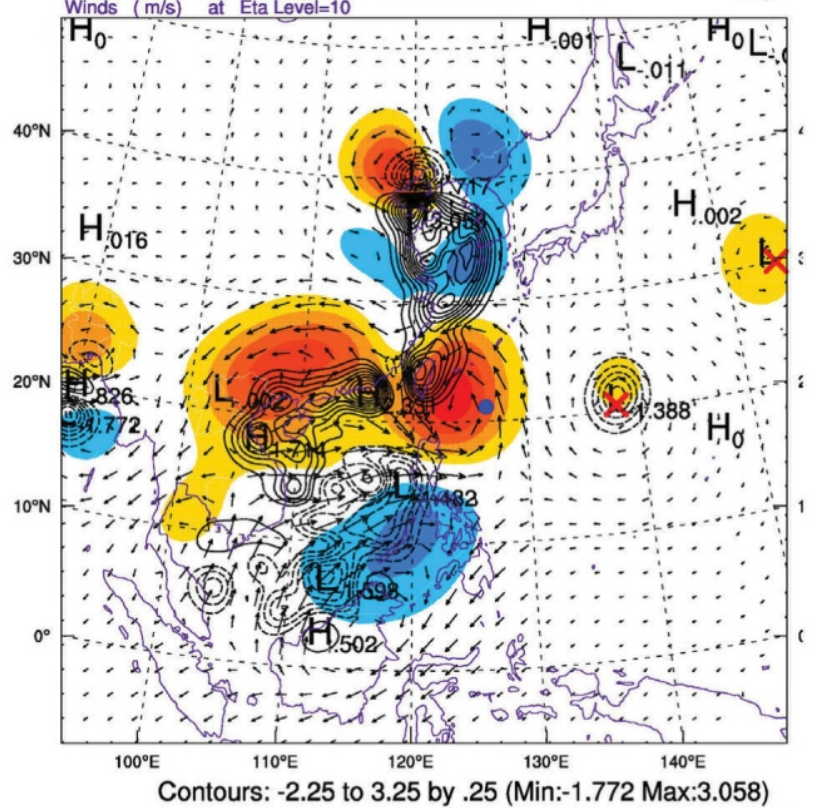

perturbation potential temperature $(\mathrm{K})$

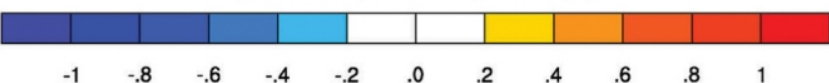

Fig. 3. The initial temperature $\left(\mathrm{K}\right.$, shaded), water vapor $\left(\mathrm{g} \mathrm{kg}^{-1}\right.$, contour), and wind ( $\mathrm{m} \mathrm{s}^{-1}$, vector) increments of EPH (a) at sigma level 0.83 ( $\left.838 \mathrm{hPa}\right)$ for domain 1. (b), (c), and (d) as in (a) but for SSMI, EPH+SSMIpw, and EPH+SSMI, respectively. The contour interval for water vapor increments is $0.25 \mathrm{~g} \mathrm{~kg}^{-1}$. The temperature increments at magnitudes larger than $0.2 \mathrm{~K}$ are shaded; the interval is $0.2 \mathrm{~K}$. The reference wind vector is indicated over the top of each panel. The locations of the two GPS soundings are marked by crosses. The initial typhoon center southeast of Taiwan is denoted by blue dot. 
perature increments and negative moisture increments for the corresponding significant impact regions surrounding the $\mathrm{RO}$ point at a radius of about $600 \mathrm{~km}$. The magnitudes of the temperature and moisture increments increase with height in the lower troposphere, reaching their maximum amounts slightly above $838 \mathrm{hPa}$. Sounding 1 produces negative moisture increments up to $-1.2 \mathrm{~g} \mathrm{~kg}^{-1}$, which is significantly larger than that of sounding 2 . However, the temperature increment (less than $0.5^{\circ} \mathrm{C}$ ) for sounding 2 is larger than that for sounding 1 . The relative changes in moisture in general are thus considerably larger than those in temperature, as a typical response to a more dominant role played by the moisture in the relative change of refractivity. The distributions of wind increment are different for the two soundings. For sounding 1, the dipole distribution of the wind increment near the surface (figure not shown) changes into an anticyclonic increment at about $838 \mathrm{hPa}$, which gives a slight strengthening of the southwestern part of the subtropical high (figures not shown). This is consistent with the aforementioned drying near the RO location of sounding 1. On the contrary, the intake of SSM/I data (denoted by SSMI in Table 1) produces larger temperature and moisture increments over the East Asia coastal area and the marginal seas. The horizontal distributions of the increments are similar at levels below $838 \mathrm{hPa}$ (figures not shown); the level near $838 \mathrm{hPa}$ is found to have larger increments for both temperature and moisture. Large positive moisture increments are produced along the coast with several local maxima (Fig. 3b). There is a local maximum of more than $1.5 \mathrm{~g} \mathrm{~kg}^{-1}$ just over Taiwan. The temperature increments are closely related to the wind increments, with two positive increments in correspondence to cyclonic wind increments at both sides of Taiwan. The cyclonic wind increment to the east is close to the initial typhoon center (marked by filled circle in Fig. 3b), which might have positive contribution to modifications on the typhoon's cyclonic circulation. On the other hand, there is also an increase in southwesterlies over the South China Sea, where larger negative moisture increments are found. It was found that the maximum wind increment can reach to about $9 \mathrm{~m} \mathrm{~s}^{-1}$ below $838 \mathrm{hPa}$ (figures not shown). However, the magnitudes of the wind increments (less than $2 \mathrm{~m} \mathrm{~s}^{-1}$ ) are reduced significantly as seen in Fig. $3 \mathrm{c}$ when only PW data are assimilated.

The ingestion of both GPS RO soundings and SSM/I data (denoted by EPH+SSMI in Table 1) produces a combination of the increment distributions of the aforementioned EPH and SSMI experiments (Fig. 3d). It should be noted that the magnitudes of increments for GPS RO soundings are considerably smaller than those for SSM/I data. Finally, combining the GPS RO soundings with PW data (denoted by EPH+SSMIpw in Table 1) induces similar moisture increments as the SSMI and EPH+SSMI experiments show (by comparing Fig. 3c with $b$ and d). However, the corresponding temperature and wind increments are quite dif- ferent from the result of the EPH+SSMI experiment. The wind increments for $\mathrm{EPH}+\mathrm{SSMIpw}$ are significantly smaller than those for EPH+SSMI. Compared with EPH, the former has resulted in weaker northeasterlies, while the latter enhances southwesterlies over the South China Sea.

Briefly, the influences of assimilated GPS RO soundings and SSM/I data on the initial fields are quite different. The GPS RO soundings may amplify the steering flow east of Typhoon Bilis at the initial time. The SSM/I data induce significant adjustments over the East Asian coastal regions, while having much weaker influences on the typhoon. The PW data tend to moisturize the lower atmosphere, while the near-surface wind speed data increase the cyclonic wind increments in the vicinity of the typhoon.

\section{MODEL RESULTS}

\subsection{Typhoon Track and Intensity}

The general performances of the experiments are examined and evaluated in terms of typhoon track and intensity prediction in this session. The simulated typhoon tracks for the five experiments, which are defined as the positions of minimum sea-level pressure, are shown in Fig. 4. The best track from CWB is also overlapped for comparisons. According to the CWB's report, Typhoon Bilis moved northwestward toward Taiwan and made landfall at I-Lan (the northeast county of Taiwan) at 1420 UTC 13 July. The typhoon center left Taiwan by 1720 UTC 13 July, moved slightly southward for the next few hours and turned northwestward toward mainland China. The typhoon then made another landfall in mainland China at about 0500 UTC 14 July.

Overall, the best track shows a rather persistent northwestward movement during the simulation period, with slight variations in the track. All the experiments produce much larger variations as compared to the CWB's best track. Without the relocation of the initial typhoon center, all the simulated initial vortex centers are slightly apart from the observed location. In the first day, the simulated tracks for the five experiments move north-northwestward to the north of the CWB's best track. All the simulated tracks then rebound back to the best track by the end of the first day and keep wandering around the east of Taiwan before $36 \mathrm{~h}$. All the simulated typhoon centers make landfall at positions to the south of the best track, except for that of EPH that passes over the northern tip of Taiwan. The simulated typhoon for the control experiment (NONE), however, exhibits a much southward-biased track over Taiwan, as compared to those of other assimilation runs and the best track. After passing by Taiwan, all simulated typhoons for the assimilation experiments move southwestward (rather than northwestward as observed) before impinging mainland China.

Regarding the possible mechanism for the improvement on track prediction with assimilation of the GPS RO 


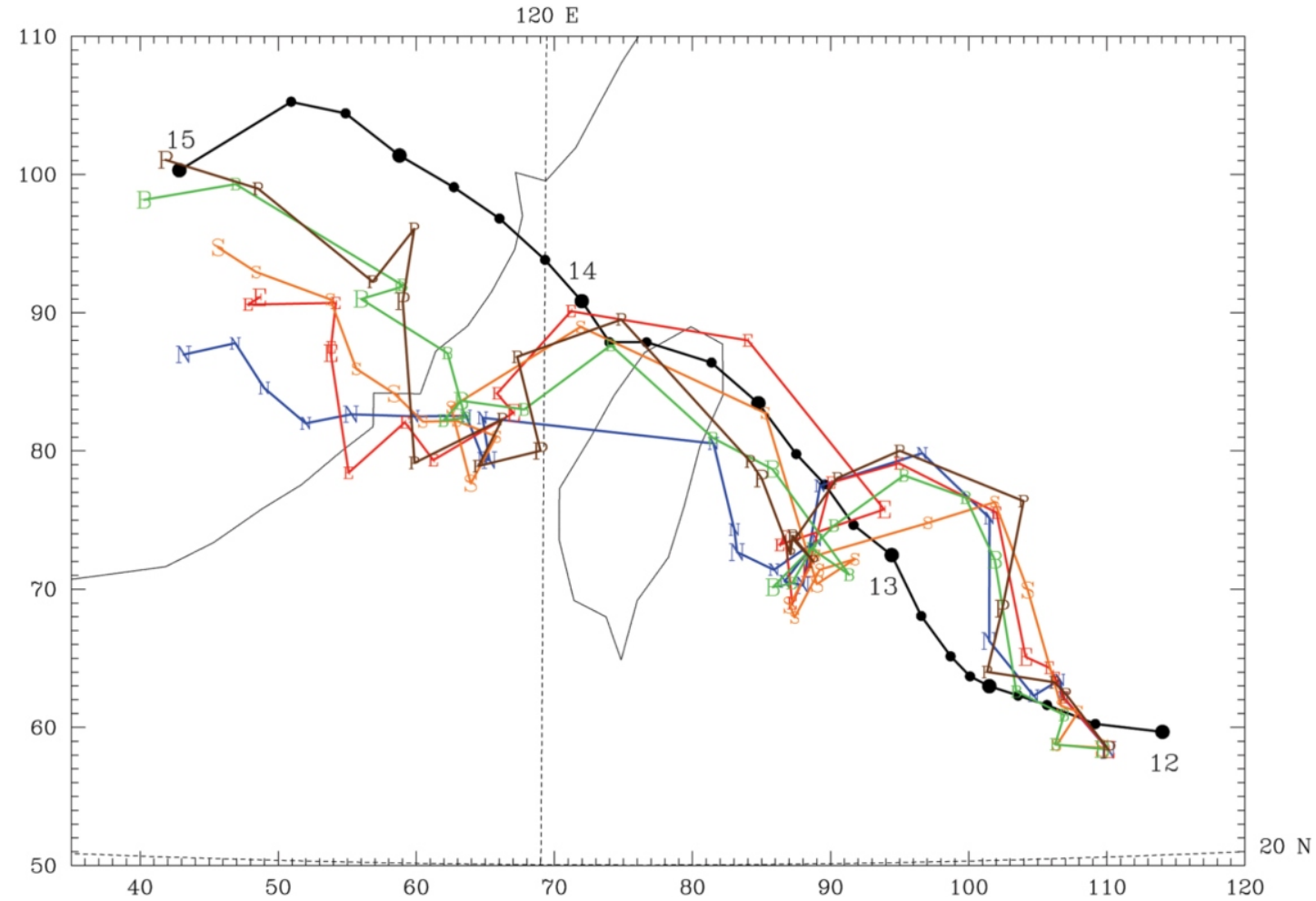

Fig. 4. The best track (denoted by dot) from the CWB and the simulated tracks for experiments of NONE, EPH, SSMI, EPH+SSMI, and EPH+SSMIpw, denoted by N (blue), E (red), S (camel), B (green), and P (brown), respectively. Cyclone centers at every 0000 and 1200 UTC are indicated by larger symbols of the experiments.

soundings, we may inspect the temporal evolutions of initial increments and the induced changes in other fields (e.g., wind). Herein, we should note that the difference in typhoon tracks may result from nonlinear interactions among the initial increments, the evolving typhoon vortex and the environmental flow. We found that more southerly wind increments in fact are induced at several earlier times to the south of the vortex core (figures not shown), which might help a more northward track as for EPH. However, such a more pronounced southerly wind component does not always exist at most of the heights and times prior to landfall. Nevertheless, the above possible mechanism may explain to some extent why the southward track detour prior to landfall can be greatly reduced in EPH.

Compared with observations, the simulated landfall for the assimilation experiments is delayed by about $3 \mathrm{~h}$. The typhoon for the control experiment makes landfall after $42 \mathrm{~h}$ (18 UTC 13 July), which is far behind the others. All the simulated typhoons move faster than the observed during the second day and pass over Taiwan in 3 hours. Finally, all simulated typhoon centers impinge the mainland China in 57 - $60 \mathrm{~h}$ (0900 - 1200 UTC 14 July), slightly later than the observed time (about $53 \mathrm{~h}$ ).

It should be noted that the simulated track for EPH well resembles the best track during $36-42 \mathrm{~h}$, in spite that it touches the northern tip of the island. For a more statistical evaluation, the 24-h average track errors for the three days are examined and are shown in Fig. 5. There are only small differences among the experiments in the first day, due to the fact that all the experiments give northward biased tracks. For the second and third days, all assimilation experiments perform better than the control experiment (NONE). The experiments with combined assimilation $(\mathrm{EPH}+\mathrm{SSMI}$ and

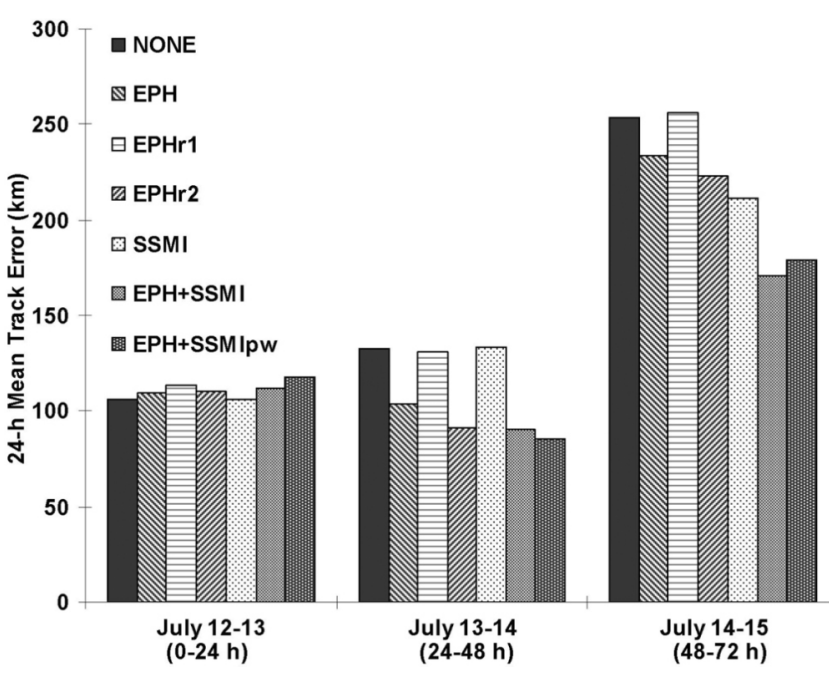

Fig. 5. Average track errors $(\mathrm{km})$ during $0-24,24-48$, and $48-72 \mathrm{~h}$ for all experiments. 
$\mathrm{EPH}+\mathrm{SSMIpw}$ ) outperform the other experiments. Consistent with Fig. 4, the control experiment gives the largest track error in this period. The track errors in general are smaller for experiments of SSM/I data with assimilation of additional GPS data. It should be pointed out that the SSM/I data alone, gives track errors as large as the control experiment for the first two days. These comparisons reveal the fact that the ingestion of the GPS RO soundings (in particular sounding 1) may play a crucial role in the improvement of track prediction.

The simulated typhoon intensities at every $3 \mathrm{~h}$ during the entire integration period are shown in Fig. 6. The simulated typhoon intensities for all the experiments are similar in trends, with larger diversity later in the simulation. Note that the initial typhoon central pressures are about $989 \mathrm{hPa}$ for the three experiments (NONE, EPH, and EPH+SSMIpw) and are weaker than the observed analysis $(985 \mathrm{hPa})$ from CWB. When the wind speed information has been assimilated in the experiments (SSMI and EPH+ SSMI), the typhoon tends to be relatively stronger at later times. The simulated typhoons for all the experiments intensify to the observed within the first $6 \mathrm{~h}$ (Fig. 6a). The intensifications on average are faster than the observed and last for about two days, while the observed has been suppressed more quickly after 0600 UTC 14 when all the simulated typhoons also begin to weaken. In general, EPH outperforms all the other experiments, showing the best agreement with the observed. On the contrary, the control experiment (NONE) exhibits more deepening after the time of diversity, possibly resulted from a more southward track. The SSMI also produces an excessive intensification which tends to be reduced by the assimilation of the GPS RO soundings. From Fig. 6b, it can be easily identified that both EPH and EPHr2 (removing sounding 2) have nearly identical results, while EPHr1 (removing sounding 1) tends to follow the control experiment (NONE). As another manifestation, the assi-

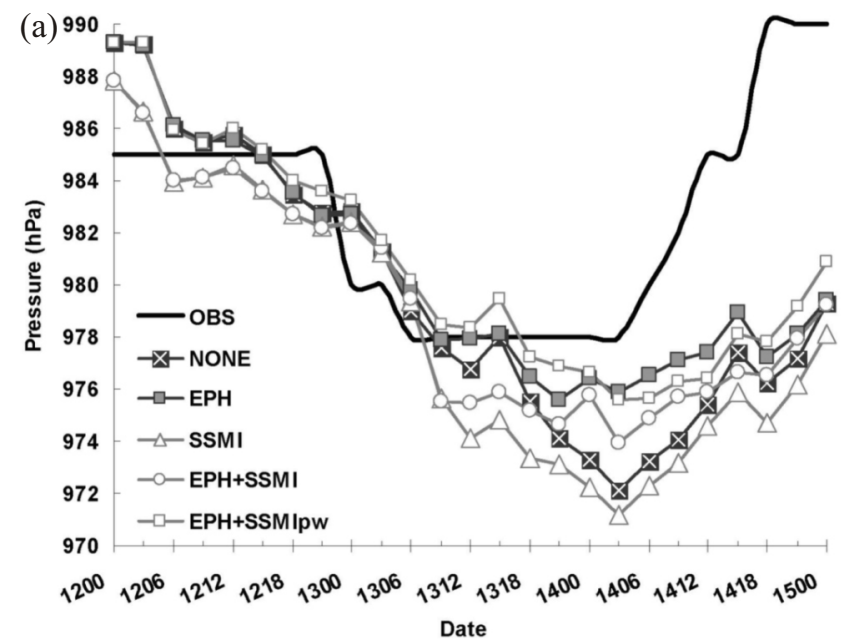

milation of the GPS RO sounding 1 is more significant for reducing the bias in track and the deepening in intensity.

\subsection{Simulated Rainfalls over Taiwan}

A subtle deviation of the simulated typhoon track, as well as the cyclone intensity, may result in appreciable differences in accumulated rainfalls over Taiwan. Evaluation on the data impact on simulated rainfall is also important for bringing out the value of the observations. For verification, we focus on the simulated rainfalls over Taiwan that can be compared and evaluated more easily against the island-wide raingauge observations.

During the first day when the Typhoon Bilis approached Taiwan, the maximum rainfall is less than $250 \mathrm{~mm}$ over northeast Taiwan (figures not shown). Typhoon Bilis then produces vigorous rainfalls during the second and third days which are distributed further south. The observed 24-h accumulated rainfalls on 13 July shows significant amounts along the western slope of the Central Mountain Range (CMR) in Taiwan, where the maximum amount of $465 \mathrm{~mm}$ is located at about $23.5^{\circ} \mathrm{N}$ (Fig. 7a). Large accumulated rainfalls are also found over the northeastern and southern Taiwan, while much smaller rainfalls appear at the eastern region of the island. After the typhoon passes over north Taiwan, tremendous rainfalls are produced over south Taiwan, with the maximum amount of $680 \mathrm{~mm}$ south of $23^{\circ} \mathrm{N}$ (Fig. 7d).

In the second day, both the control experiment (NONE) and EPH exhibit over-predicted rainfalls over south Taiwan (Figs. 7b, c). However, EPH is able to pick up some observed signal of larger rainfall near the central Taiwan, while it is unpredicted by NONE. On the other hand, the control experiment better predicts the observed rainfalls over northeast Taiwan, while EPH has considerably underestimated. Both experiments fail to reproduce major rainfalls over the

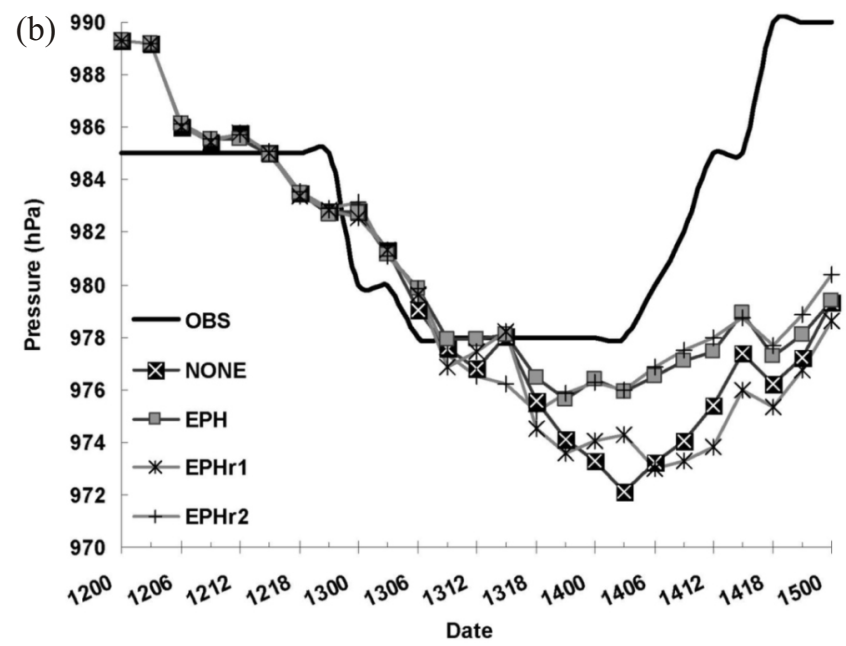

Fig. 6. Typhoon intensities (hPa) at every $3 \mathrm{~h}$ for all experiments. 
(a)

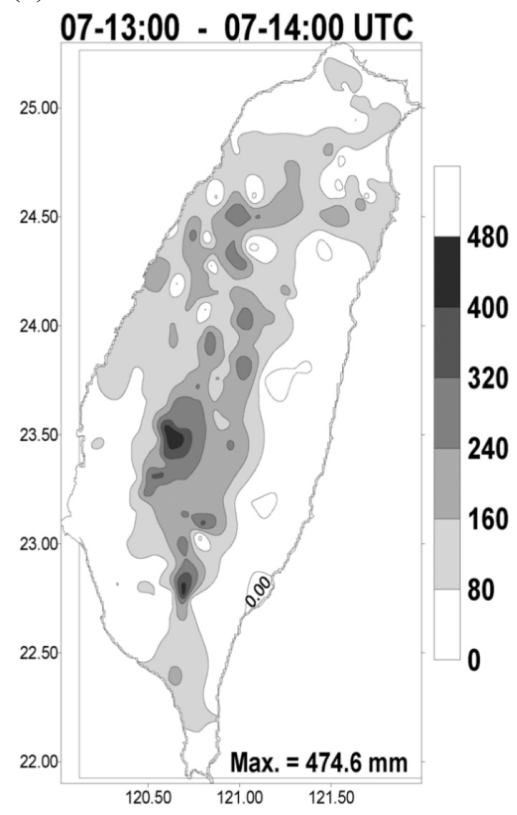

(d) 07-14:00 - 07-15:00 UTC

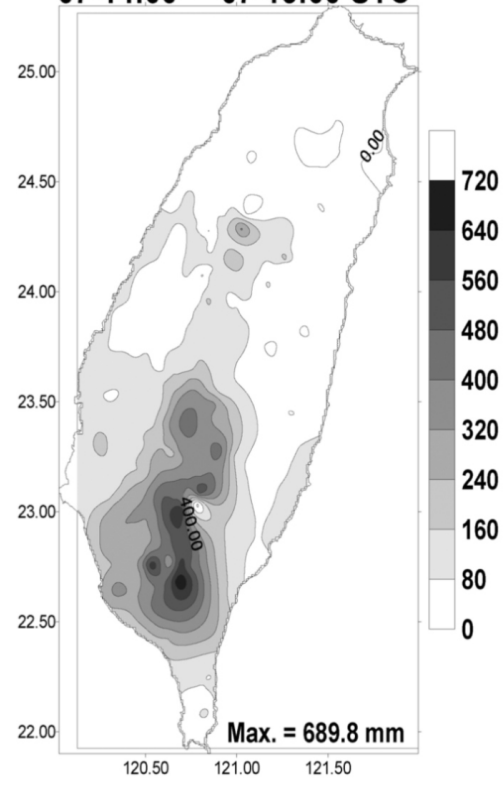

(b) Acc. Precipitation Valid; 2006-07-14_00:00:00

from 2006-07-13 00:00:00 to 2006--07-14 00:00:0

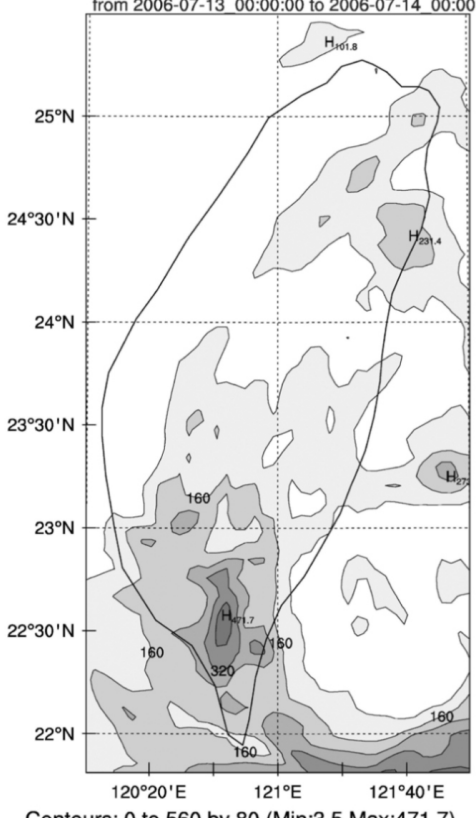

Contours: 0 to 560 by 80 (Min:3.5 Max:471.7)

(e) Acc. Precipitation Valid:2006-07-15_00:00:00
from 2006-07-14 00:00:00 to 2006-07-15 00:00:0r

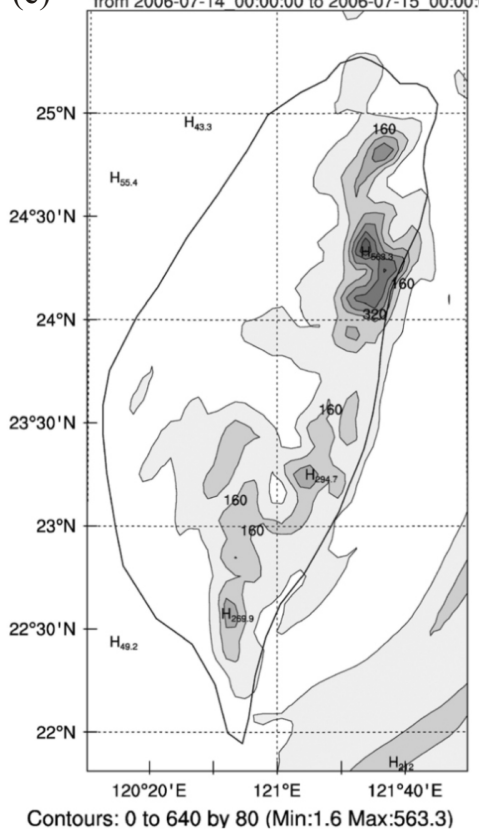

(c) Acc. Precipitation Valid:2006-07-14_00:00:00

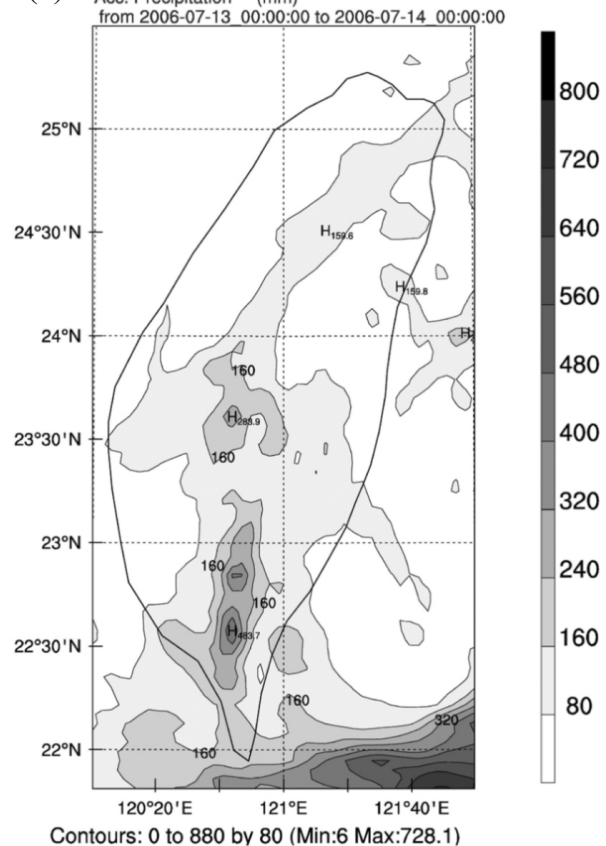

Acc. Precipitation Valid:2006-07-15_00:00:00

(f) from 2006-07-14 00:00:00 to 2006-07-15 00:00:00

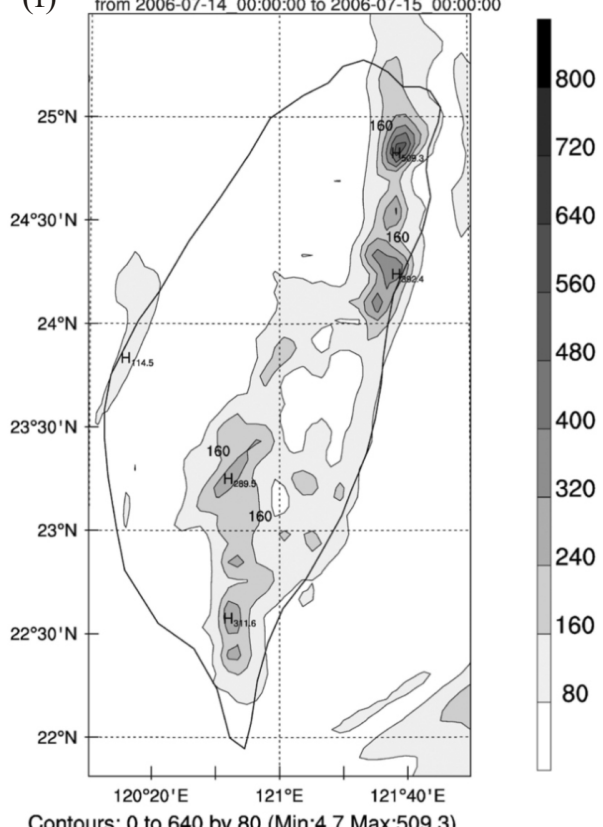

Fig. 7. The observed (a) and simulated rainfall for experiments of (b) NONE and (c) EPH at domain 3 during 24 - 48 h. (d), (e), and (f) as in (a), (b), and (c), respectively, but during $48-72 \mathrm{~h}$. The contour interval is $80 \mathrm{~mm}$.

central coastal area. For the third day, both experiments reproduce some of the observed intense rainfalls over the far south of Taiwan, while larger rainfalls are obtained by EPH in better agreement with the observations (Figs. 7e, f). There is less rainfall along the eastern coast for EPH as compared to the control experiment and is also in better agreement with the observations. However, both experiments produce significant rainfalls over northeast Taiwan greatly differing from the observations, probably due to their southward biased tracks at later times.

In brief, the EPH experiment shows a marked improvement in simulation of island's rainfall. To understand why such an improvement can be produced, we show the sealevel pressure and near-surface wind in the innermost domain in Fig. 8. At 36 h, the simulated central low of the control experiment is located slightly to the south of that in the 
(a)

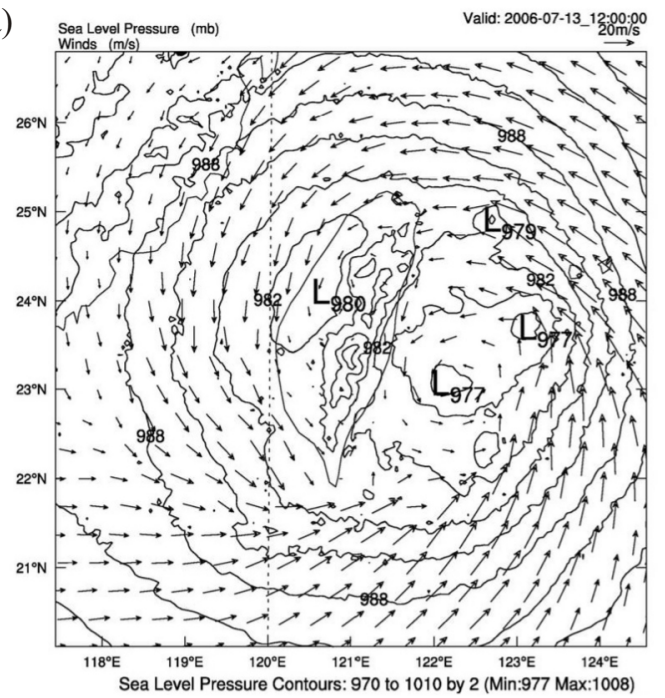

(c)

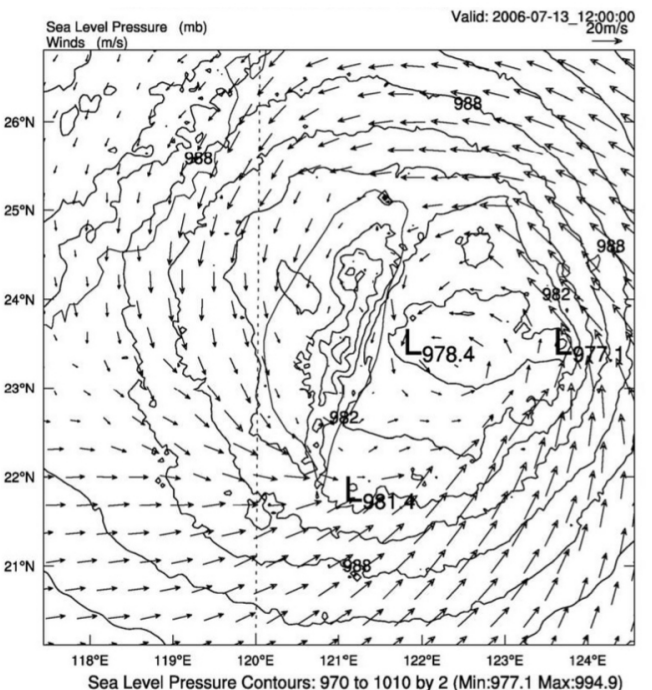

(e)

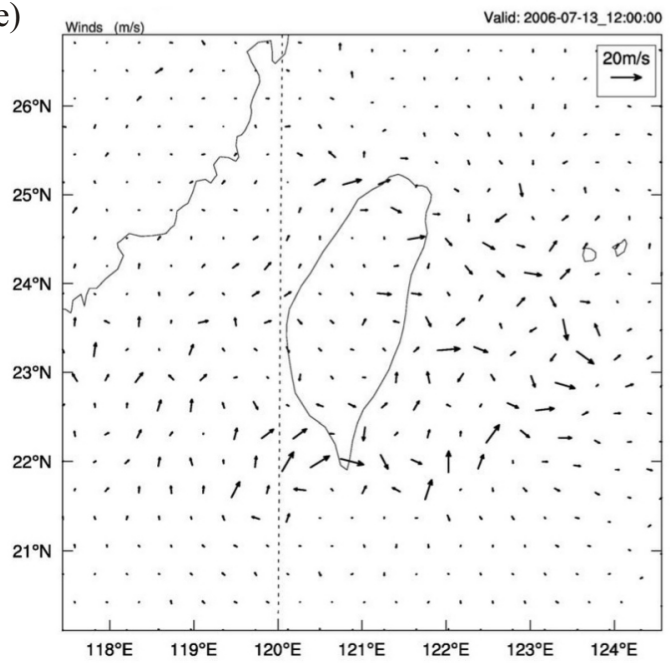

(b)

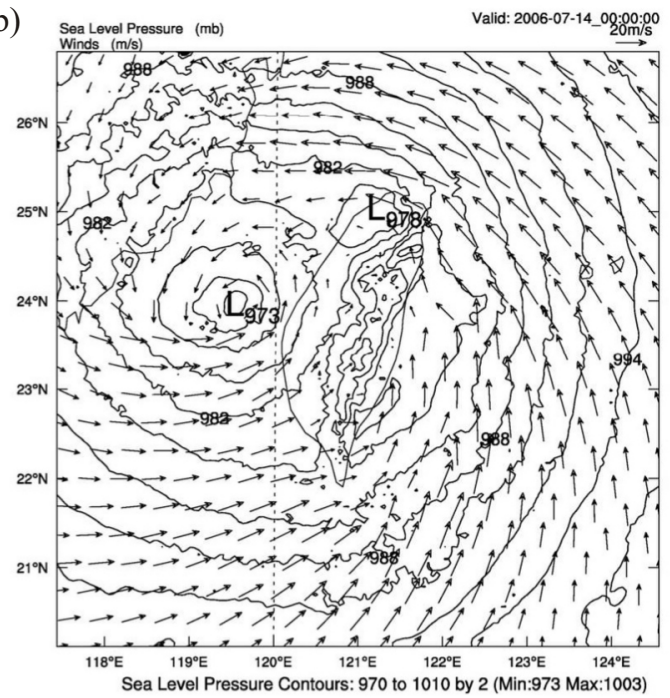

(d)

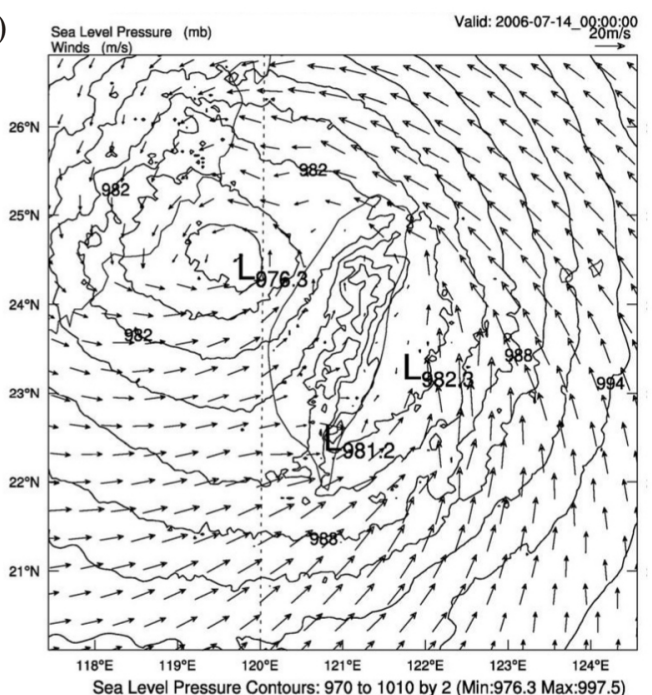

(f)

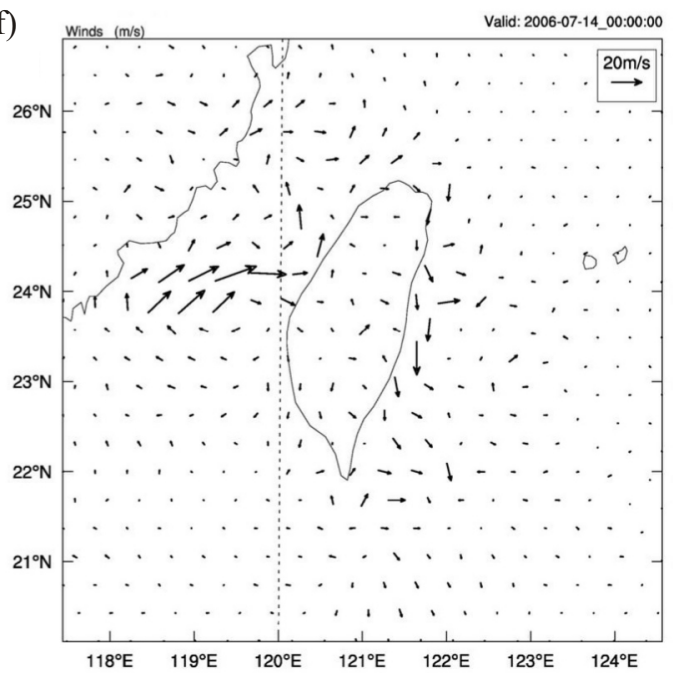

Fig. 8. Simulated sea level pressure (hPa) and near-surface wind $\left(\mathrm{m} \mathrm{s}^{-1}\right)$ at domain 3 at (a) $36 \mathrm{~h}$ and (b) $48 \mathrm{~h}$ for experiment NONE. (c) and (d) as in (a) and (b), but for experiment EPH, respectively. Near-surface wind differences ( $\mathrm{m} \mathrm{s}^{-1}$ ) at domain 3 at (e) $36 \mathrm{~h}$ and (f) $48 \mathrm{~h}$ for experiment EPH (EPH minus NONE). The contour interval is $2 \mathrm{hPa}$. The reference wind vector is indicated over the top of each panel. 
experiment EPH (Figs. 8a, c). The vortex intensifies for the next $12 \mathrm{~h}$ while passing over the central Taiwan, and is still located to the south of that for EPH (Figs. 8b, d). During this period, the simulated central low of the experiment EPH is closer to the observed, resulting in stronger southwesterlies and westerlies toward west Taiwan (Figs. 8e, f) and thus producing the larger rainfalls over the central Taiwan in the second day. We found that the major rainfalls over northeast Taiwan in the second day are better simulated by the control experiment than the experiment EPH. However, the improvement is at the cost of the rainfall prediction over the central Taiwan, and the northeastern major rainfall remains to prevail in the third day, which is not supported by observations.

The rainfall predictions are improved when the SSM/I data have been assimilated. In the second day, all three experiments using SSM/I data well simulate the intense rainfalls over south Taiwan (Figs. 9a-c). Moreover, EPH+ (a) Acc. Precipitation Valid:2006-07-14_00:00:00

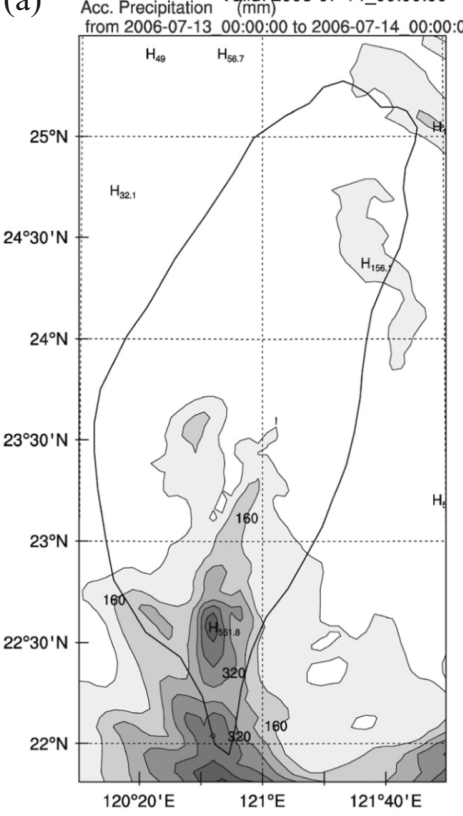

Contours: 0 to 640 by 80 (Min:.6 Max:576.1)

(d) Acc. Precipitation Valid:2006-07-15_00:00:00

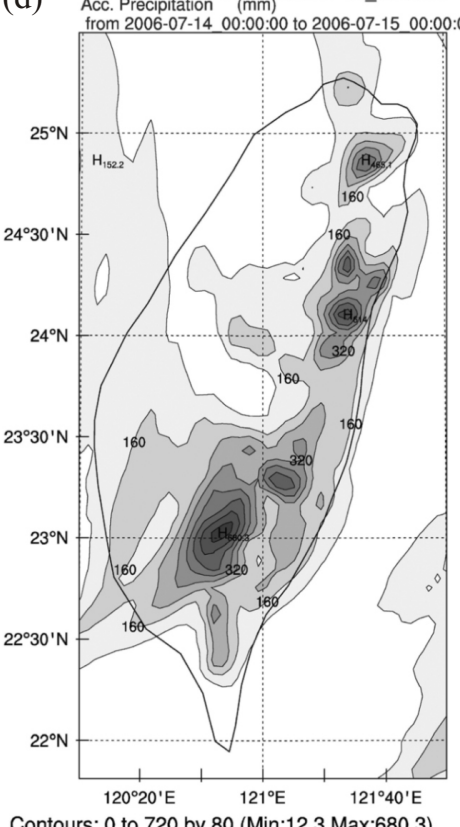

(b) Valid:2006-07-14_00:00:00

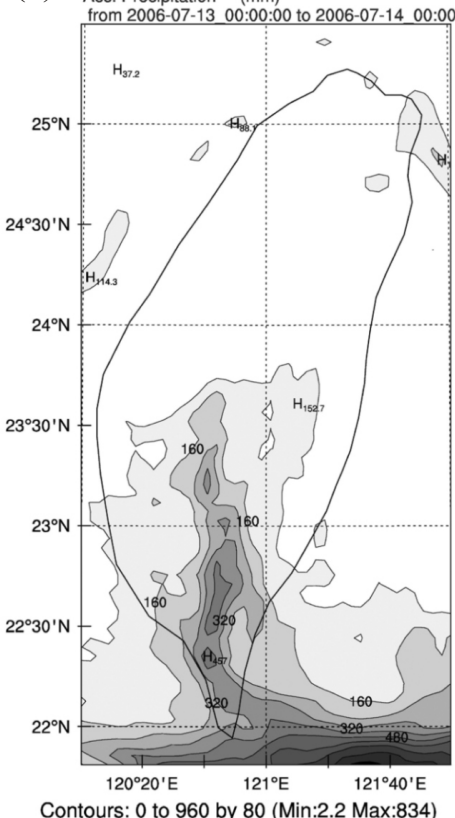

(e) Acc. Precipitation Validid:2006-07-15_00:00:00

Acc. Precipitation
from 2006-07-14 00:00:00 to 2006-07-15 00:00:00

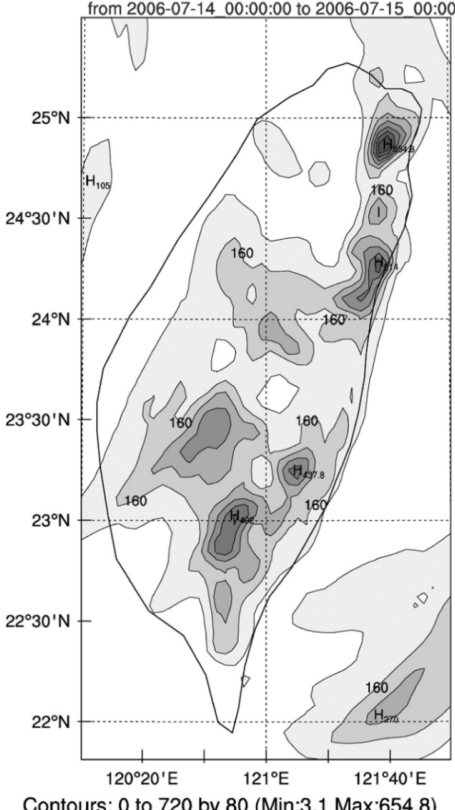

(c) Acc. Precipitation Valid:2006-07-14_00:00:00

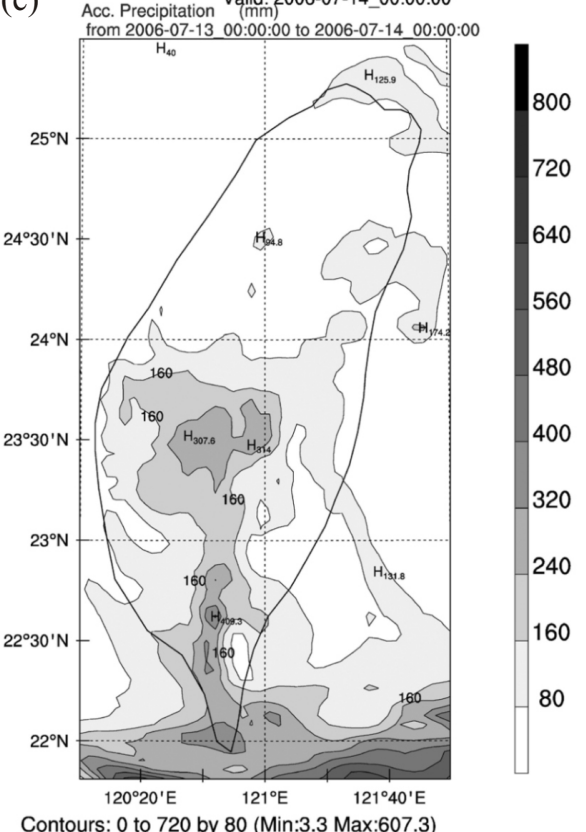

Contours: 0 to 720 by 80 (Min:3.3 Max:607.3)

(f) Acc. Precipitation Valid: 2006-07-15_00:00:00

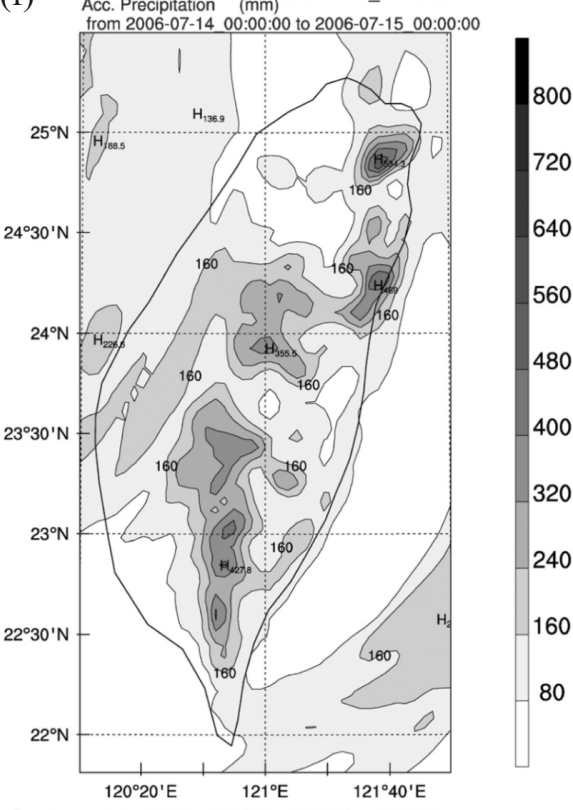

Contours: 0 to 640 by 80 (Min:10.2 Max:534.3)
720

640

560

480

400

320

240

60

80

Fig. 9. Simulated rainfalls at domain 3 during 24 - $48 \mathrm{~h}$ for experiments (a) SSMI, (b) EPH+SSMI, and (c) EPH+SSMIpw. (d), (e), and (f) as in (a), (b), and (c), respectively, but during $48-72 \mathrm{~h}$. The contour interval is $80 \mathrm{~mm}$. 
SSMIpw experiment well predicts the major rainfall over central Taiwan (Fig. 9c) and outperforms the others. For the third day, these three experiments outperform the other two experiments without SSM/I data assimilation (Figs. 9d - f) in terms of the more pronounced rainfall at south Taiwan. The experiments $\mathrm{EPH}+\mathrm{SSMI}$ and $\mathrm{EPH}+\mathrm{SSMIpw}$ also predict more intense rainfall over the central Taiwan, agreeing better with the observations (Fig. 7d). Overall, the assimilation with SSM/I data leads to improvement in predication of major rainfall over Taiwan, which is further improved by additional GPS data assimilation (e.g., comparing Figs. 9a, d with Figs. 9b, e or Figs. 9c, f).

\subsection{Sensitivity Tests on Individual GPS Soundings}

The previous comparisons demonstrate the importance of the GPS soundings in improving simulated typhoon track as well as major rainfall over Taiwan. It is possible to identify the sole impact of each individual sounding by conducting two sensitivities experiments (EPHr1 and EPHr2) with removal of each sounding. Figure 10 shows the simulated typhoon tracks for the two sensitivity tests; the simulated tracks for the control experiment and EPH are also included for comparisons. The simulated track for EPHr2 (removing sounding 2 near the model boundary) is closer to the best track, while EPHr1 (removing sounding 1 nearer the typhoon) has a similar perfor- mance compared to that of the control experiment. The track errors for EPHr2 are even slightly smaller than for EPH as indicated by the 24-h average track errors (Fig. 5). As seen, EPHr2 produces an over-prediction of rainfall over south Taiwan at the second day (Fig. 11b), which is, further overestimated by EPHr1 (Fig. 11a). For the third day, EPHr2 gives better more intense rainfall near the southern end of Taiwan in approximation to EPH (Figs. 11d), while the EPHr1 is closer to the control experiment, both producing similar weaker rainfall (Figs. 7e and 11c).

\subsection{Evaluation of Model Performances}

Although the previous comparisons reveal some positive impacts of GPS RO soundings on the simulation of Typhoon Bilis, it is desirable to evaluate the model performances more objectively. The threat score (TS) is adopted to verify the rainfall prediction for each experiment. The TS for each rainfall threshold is defined as:

$T S=\frac{H}{F+O-H}$

where $H$ is the number of the grids on which both the simulated and the observed rainfalls exceed the threshold, $F$ and $O$ are the number of the grids on which the simulated and

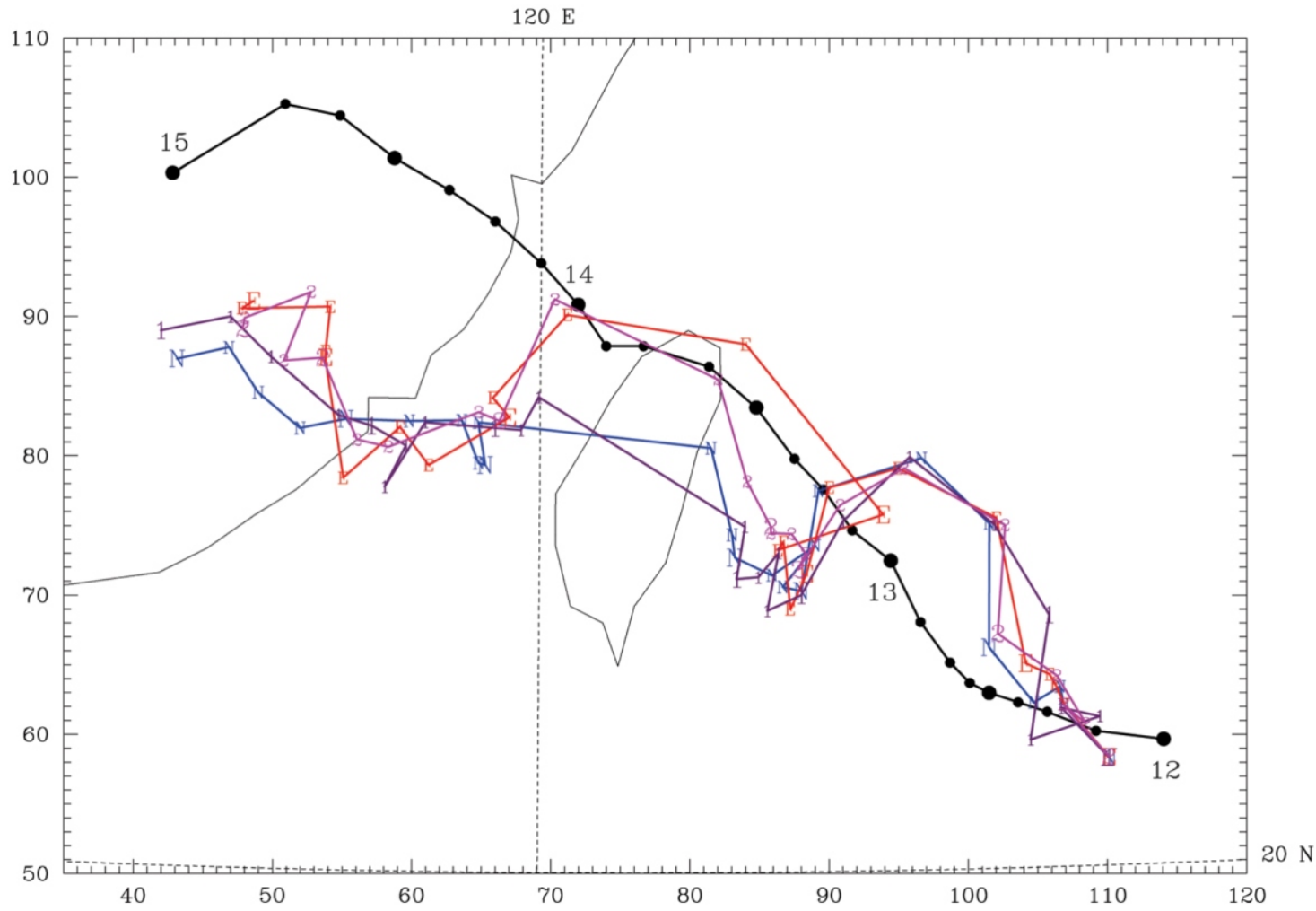

Fig. 10. The same as in Fig. 3 but for experiments NONE, EPH, EPHr1, and EPHr2 denoted by N (blue), E (red), 1 (purple), and 2 (violet), respectively. 
(a) Acc. Precipitation Valid:2006-07-14_00:00:00 Acc. Precipitation (mm)
from 2006-07-13 00:00:00 to 2006-07-14 00:00:00

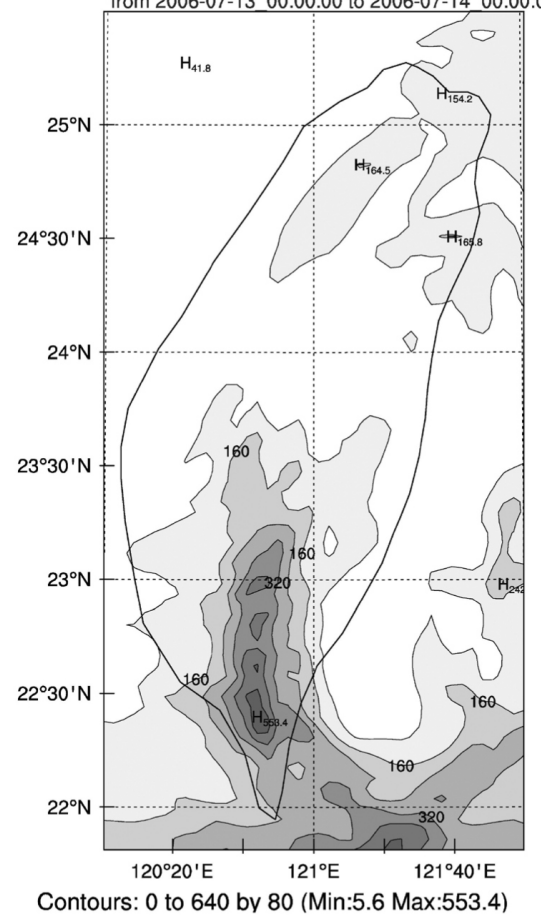

(c) Acc. Precipitation Valid:2006-07-15_00:00:00 from 2006-07-14 00:00:00 to 2006-07-15 00:00:00

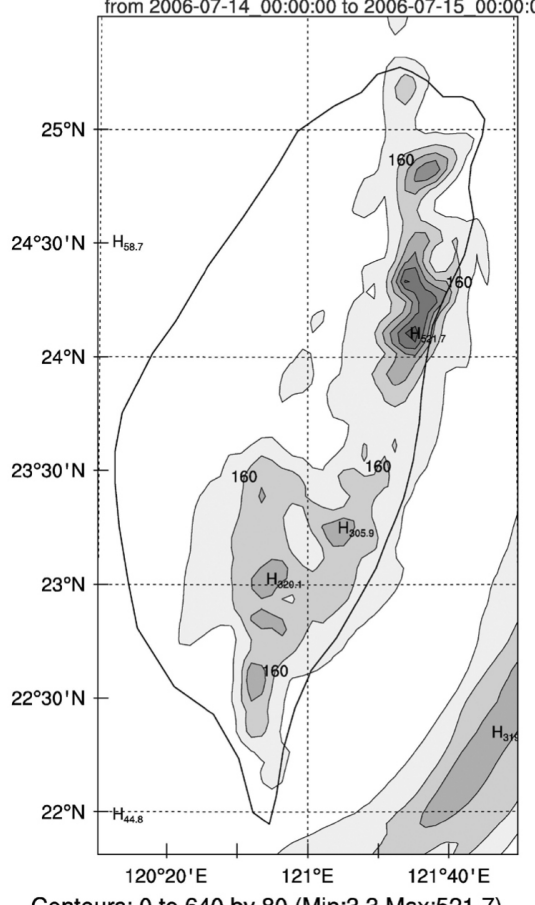

(b) Acc. Precipitation Valid: 2006-07-14_00:00:00

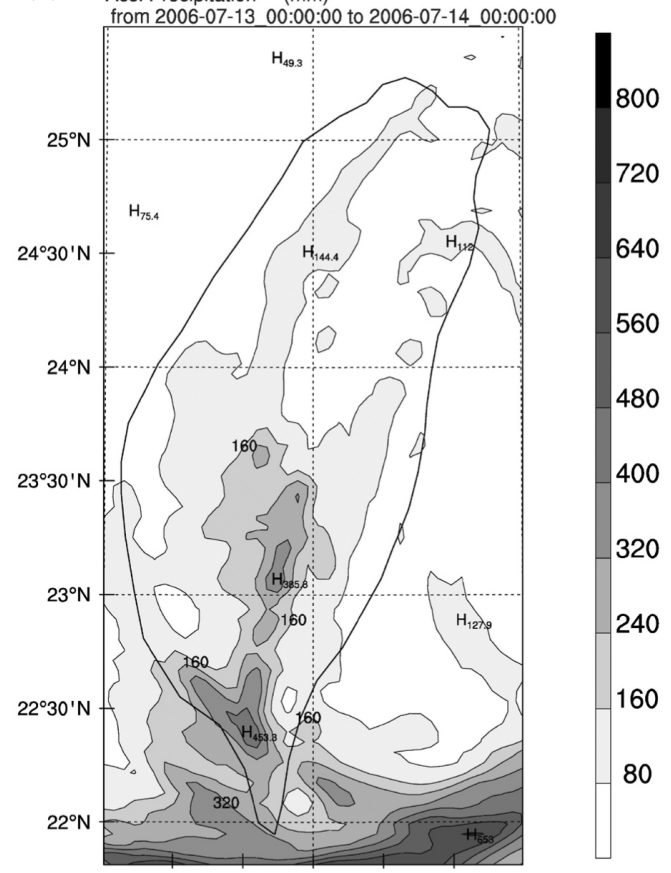

$120^{\circ} 20^{\prime} \mathrm{E} \quad 121^{\circ} \mathrm{E} \quad 121^{\circ} 40^{\prime} \mathrm{E}$

Contours: 0 to 720 by 80 (Min:4.8 Max:653)

(d) Acc. Precipitation Valid:2006-07-15_00:00:00

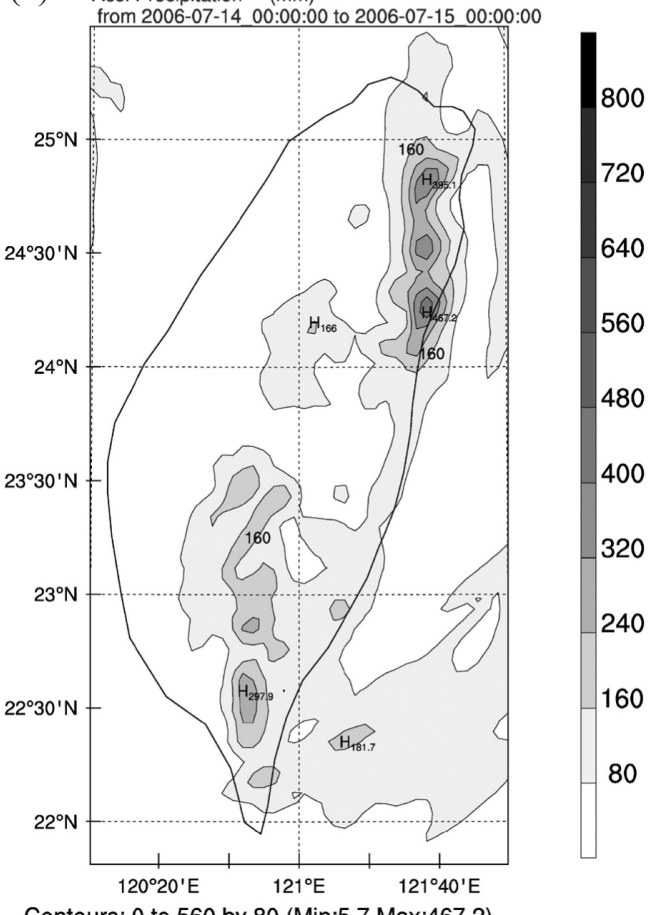

Contours: 0 to 560 by 80 (Min:5.7 Max:467.2)

Fig. 11. Simulated rainfalls at domain 3 during 24 - 48 h for experiments (a) EPHr1, and (b) EPHr2. (c) and (d) as in (a) and (b), respectively, but during $48-72 \mathrm{~h}$. The contour interval is $80 \mathrm{~mm}$.

observed rainfalls exceed the threshold, respectively. To perform the TS calculation, the station data are first interpolated into the finest domain using Cressman scheme with an influential weighting radius of $20 \mathrm{~km}$. TS for each threshold is then computed by using the model results and the objectively analyzed observations.

Figure 12 gives the TSs with thresholds of $1,5,10,15$, 20,50 , and $100 \mathrm{~mm}$ for $0-24,24-48$, and $48-72 \mathrm{~h}$ accumu- 
(a)

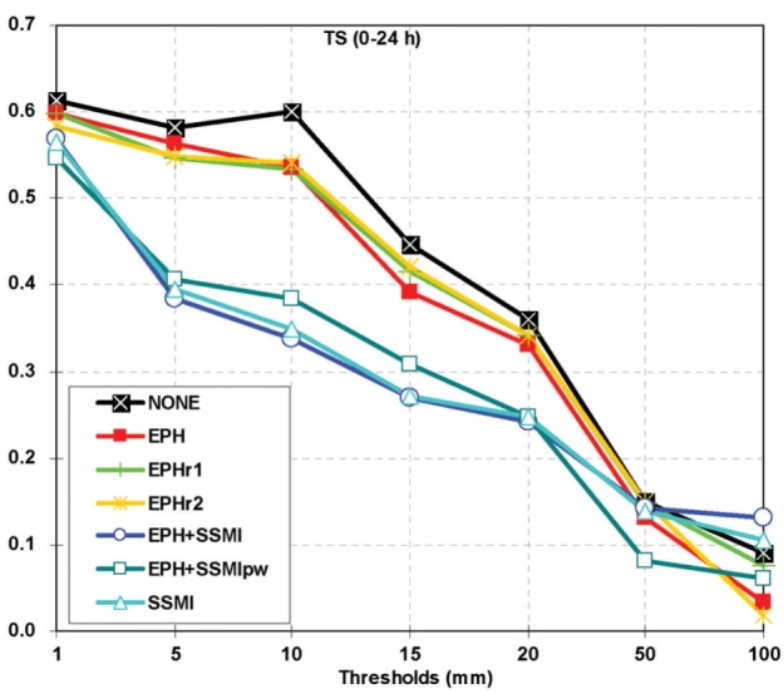

(b)

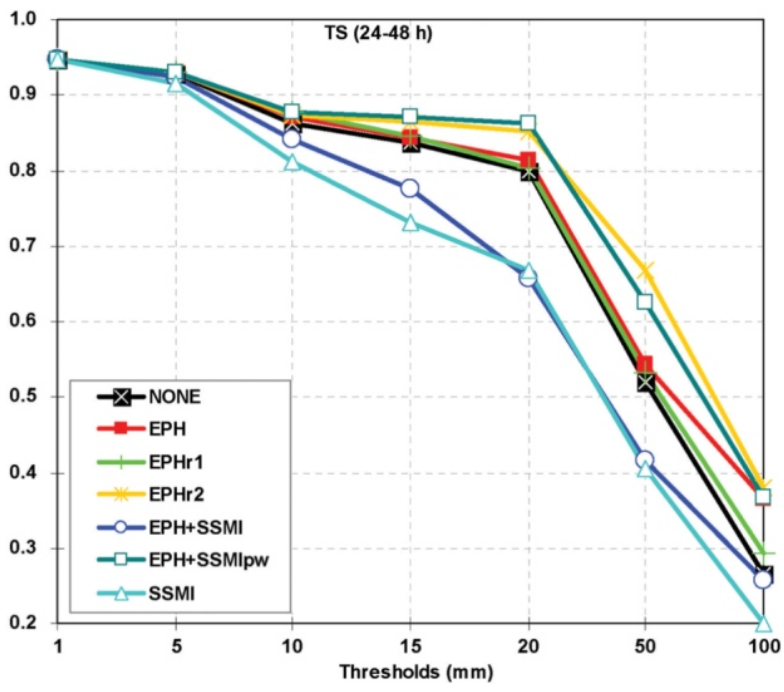

(c)

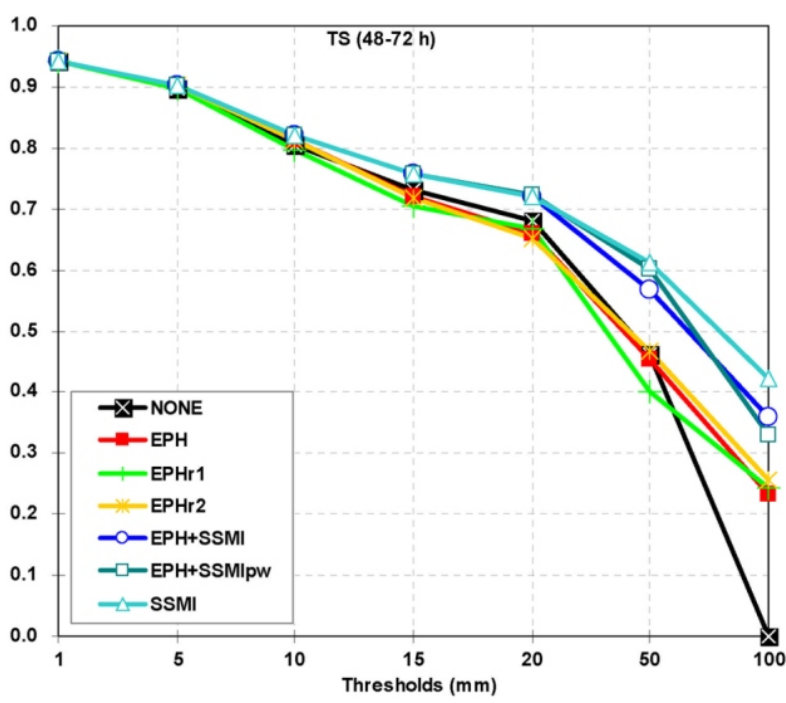

Fig. 12. The threat scores with thresholds of 1, 5, 10, 15, 20, 50, and $100 \mathrm{~mm}$ for the accumulated rainfalls during (a) $0-24 \mathrm{~h}$, (b) $24-48 \mathrm{~h}$, and (c) $48-72 \mathrm{~h}$ for all experiments. lated rainfalls for all experiments. In general, TS is higher for smaller thresholds and gradually decreases with increased threshold. The control experiment has the best performance for smaller thresholds in the first day (Fig. 12a). Experiments only with the assimilation of GPS RO soundings have obtained higher scores than those with SSM/I data, except for the higher thresholds (e.g., 50 and $100 \mathrm{~mm}$ ). For thresholds of 10 and $15 \mathrm{~mm}$, the scores for EPH+SSMIpw (EPH with additional PW data) are slightly higher than the experiments SSMI and EPH+SSMI.

In the second day, EPHr2 and EPH+SSMIpw obtain the higher scores for thresholds in $10-100 \mathrm{~mm}$ (Fig. 12b). The impact of the GPS RO sounding 1 is competitive to the combined impact of the two GPS RO soundings and PW data. For less intense rainfalls, both EPHr1 and EPH perform equally well as compared to the control experiment. However, for the high threshold (100 mm), EPH and EPHr2 give the higher TS, indicating the influence of GPS RO sounding 1. Experiments SSMI and EPH+SSMI obtain relatively lower TSs than the other experiments. Ingestion of the wind speed data thus has not improved the rainfall simulation in Taiwan. For the third day, however, all the experiments with the SSM/I data assimilation give higher TSs for thresholds larger than $10 \mathrm{~mm}$ (Fig. 12c), which is consistent with the better distributions of major rainfall in Fig. 9. The improvement in prediction of more intense rainfall emerging at later times may be attributed to reduced average track errors (Fig. 5) at this day for the experiments with SSM/I data assimilated.

\section{CONCLUSIONS}

The present study aims at exploring the impact of the GPS RO refractivity soundings from FORMOSAT-3 on typhoons impinging Taiwan. We have carefully selected the simulation of Typhoon Bilis that made landfall in Taiwan in July 2006. Luckily in this case, RO soundings were located in favorable regions for tracking the storm's movement. The WRF model (version 2.1.2) and the new nonlocal operator for GPS RO soundings (Chen et al. 2009) are applied for this study. The new operator assimilates the integrated GPS refractivity along the straight raypath, defined as excess phase (EPH), and thus may take into account the effect of horizontal gradient. The model performances are evaluated in terms of comparisons on typhoon track and intensity, and the associated rainfalls over Taiwan. In addition, on top of available precipitable water (PW) and near-surface wind speed retrieved from SSM/I data, we have also explored the impact of the combined assimilation on model prediction. For this case, there are two GPS soundings within the outermost domain near the model initial time, one to the east of the typhoon vortex (sounding 1) and the other to the southwest of the subtropical high (sounding 2), while the SSM/I 
data provide a swath passing over the East Asia coastal regions west of the typhoon center.

The initial increments after assimilation indicate that the GPS RO refractivity soundings contribute most to moisture increments (up to $-1.2 \mathrm{~g} \mathrm{~kg}^{-1}$ ), with dryness occurring in the lower troposphere corresponding to the occultation positions. The assimilation of PW induces much larger and wider moisture increments to the west of the typhoon center. The ingestion of oceanic near-surface wind speeds produces more pronounced temperature and wind increments at lower levels. The ingestion of SSM/I wind speed results in cyclonic wind increments in the vicinity of the typhoon, and enhanced southwesterlies to the south at lower levels.

The SSM/I data improve the predictions of typhoon intensity and intense rainfall over Taiwan, especially for the later simulation time, but generally have a less impact on track prediction as compared to the GPS RO data. Assimilation of the two GPS RO soundings leads to a marked improvement on the track that otherwise will be southward biased prior to landfall, and also enhances the positive impact when the SSM/I data have been assimilated as well. The improvement using the GPS RO soundings is also found on daily rainfall but only for larger thresholds at later times when the bias in track in the control experiment is remedied. In general, the combined assimilation of both GPS RO soundings and SSM/I data has shown the most promising performance. Finally, the sensitivity tests indicate that the closer GPS RO sounding 1 to the east of the typhoon plays a more crucial role in the positive impact on track prediction.

We are fortunate to be able to utilize the case of Typhoon Bilis that well illustrates the positive impact of one GPS RO sounding on typhoon track and rainfall predictions. As is well understood, typhoon prediction can be influenced by many factors, including employment of physical parameterization schemes as well as vortex bogussing. The effect of an initially imposed bogus vortex on model prediction has been investigated in the companion paper of Chen et at. (2009). Herein, we want to purify the impact of the GPS RO soundings if these do make contributions to any improvement. Such an improvement is better marked on track prediction as the southward biased track has been remedied by assimilation of GPS RO sounding 1 . Our experiments clearly demonstrate that the sounding on the conveying path of subtropical high may play a crucial role in modulating the delicate change in typhoon's movement. This subtle impact totally relies on the accumulated influences when the initial increments produced by initial GPS data assimilation take time to develop into the environment providing varying steering of the typhoon. In addition to the SSM/I data assimilation, this consolidated impact has been only slightly impeded due to their sharing influences at different upstream and downstream regions. As just mentioned, such a positive impact may be affected by many factors involved in typhoon's evolution and is beyond the scope of this study to address many relevant issues regarding the uncertainty in typhoon prediction. Nevertheless, this study provides a successful example of the impact of few GPS soundings on severe weather prediction. We are looking forward to seeing more such examples in the near future.

Acknowledgements We thank the two anonymous reviewers for their constructive comments. This study is supported by the National Science Council in Taiwan under Grants NSC 95-2111-M-008-004-AP1 and by the National Space Program Organization in Taiwan under Grants 96NSPO(B)-SP-FA07-02(B).

\section{REFERENCES}

Barker, D., W. Huang, Y. R. Guo, and A. Bourgeois, 2003: A Three-Dimensional Variational (3DVAR) Data Assimilation System for Use with MM5. NCAR Technical Note $N C A R / T N-453+S T R, 68 \mathrm{pp}$.

Barker, D., W. Huang, Y. R. Guo, A. J. Bourgeois, and Q. N. Xiao. 2004: A three-dimensional variational data assimilation system for MM5: Implementation and initial results. Mon. Wea. Rev., 132, 897-914, doi: 10.1175/ 1520-0493(2004)132<0897:ATVDAS >2.0.CO;2. [Link]

Chen, S. H. and W. Y. Sun, 2002: A one-dimensional timedependent cloud model. J. Meteorol. Soc. Jpn., 80, 99118, doi: 10.2151/jmsj.80.99. [Link]

Chen, S. H., F. Vandenberghe, G. W. Petty, and J. F. Bresch, 2004: Application of SSM/I data to a hurricane simulation. Quart. J. Roy. Meteor. Soc., 130, 801-825, doi: 10.1256/ qj.02.67. [Link]

Chen, S. Y., C. Y. Huang, Y. H. Kuo, Y. R. Guo, and S. Sokolovskiy, 2009: Assimilation of GPS refractivity from FORMOSAT-3/COSMIC using a nonlocal operator with WRF 3DVAR and its impact on the prediction of a typhoon event. Terr. Atmos. Ocean. Sci., 20, 133-154, doi: 10.3319/ TAO.2007.11.29.01(F3C). [Link]

Cucurull, L., J. C. Derber, R. Treadon, and R. J. Purser, 2007: Assimilation of global positioning system radio occultation observations into NCEP's global data assimilation system. Mon. Wea. Rev., 135, 3174-3193, doi: 10.1175/ MWR3461.1. [Link]

Healy, S. B. and J. N. Thépaut, 2006: Assimilation experiments with CHAMP GPS radio occultation measurements. Quart. J. Roy. Meteor. Soc., 132, 605-623, doi: 10.1256/qj.04.182. [Link]

Hollinger, J., 1989: DMSP Special Sensor Microwave/Imager Calibration/Validation. Final Report, Volume I, Naval Research Laboratory, Washington, DC, USA.

Hong, S. Y., Y. Noh, and J. Dudhia, 2006: A new vertical diffusion package with explicit treatment of entrainment processes. Mon. Wea. Rev., 134, 2318-2341, doi: 10.1175/ MWR3199.1. [Link]

Huang, C. Y., Y. H. Kuo, S. H. Chen, and F. Vandenberghe, 2005: Improvements in typhoon forecasts with assimilated 
GPS occultation refractivity. Weather Forecast., 20, 931953, doi: 10.1175/WAF874.1. [Link]

Kain, J. S., 2004: The Kain-Fritsch convective parameterization: An update. J. Appl. Meteorol., 43, 170-181, doi: 10.1175/1520-0450(2004)043<0170:TKCPAU>2.0.CO;2. [Link]

Kuo, Y. H., X. Zou, and Y. R. Guo, 1996: Variational assimilation of precipitable water using a nonhydrostatic mesoscale adjoint model. Part I: Moisture retrieval and sensitivity experiments. Mon. Wea. Rev., 124, 122-147, doi: 10.1175/1520-0493(1996)124<0122:VAOPWU>2.0.CO; 2. [Link]

Kuo, Y. H., X. Zou, and W. Huang, 1997: The impact of GPS data on the prediction of an extratropical cyclone: An observing system simulation experiment. Dyn. Atmos. Oceans, 27, 439-470, doi: 10.1016/S0377-0265(97)00023-7. [Link]

Kuo, Y. H., S. V. Sokolovskiy, R. A. Anthes, and F. Vandenberghe, 2000: Assimilation of GPS radio occultation data for numerical weather prediction. Terr. Atmos. Ocean. Sci., 11, 157-186.

Kuo, Y. H., T. K. Wee, S. Sokolovskiy, C. Rocken, W. Schreiner, D. Hunt, and R. A. Anthes, 2004: Inversion and error estimation of GPS radio occultation data. J. Meteorol. Soc. Jpn., 82, 507-531, doi: 10.2151/jmsj.2004.507. [Link]

Kuo, Y. H., W. S. Schreiner, J. Wang, D. L. Rossiter, and Y. Zhang, 2005: Comparison of GPS radio occultation soundings with radiosondes. Geophys. Res. Lett., 32, L05817, doi: 10.1029/2004GL021443. [Link]

Liu, H. and X. Zou, 2003: Improvements to GPS radio occultation ray-tracing model and their impacts on assimilation of bending angle. J. Geophys. Res., 108, 4548, doi: 10.1029/2002JD003160. [Link]

Rocken, C., R. Anthes, M. Exner, D. Hunt, S. Sokolovskiy, R. Ware, M. Gorbunov, W. Schreiner, D. Feng, B. Herman, Y. Kuo, and X. Zou, 1997: Analysis and validation of GPS/ MET data in the neutral atmosphere. J. Geophys. Res., 102, 29849-29866, doi: 10.1029/97JD02400. [Link]
Rocken, C., Y. H. Kuo, W. S. Schreiner, D. Hunt, S. Sokolovskiy, and C. McCormick, 2000: COSMIC system description. Terr. Atmos. Ocean. Sci., 11, 21-52.

Skamarock, W. C., J. B. Klemp, J. Dudhia, D. O. Gill, D. M. Barker, W. Wang, and J. G. Powers, 2005: A Description of the Advanced Research WRF Version 2. NCAR technical note NCAR/TN-468+STR, $100 \mathrm{pp}$.

Sokolovskiy, S., Y. H. Kuo, and W. Wang, 2005a: Assessing the accuracy of linearized observation operator for assimilation of the Abel-retrieved refractivity: Case simulation with high-resolution weather model. Mon. Wea. Rev., 133, 2200-2212, doi: 10.1175/MWR2948.1. [Link]

Sokolovskiy, S., Y. H. Kuo, and W. Wang, 2005b: Evaluation of a linear phase observation operator with CHAMP radio occultation data and high-resolution regional analysis. Mon. Wea. Rev., 133, 3053-3059, doi: 10.1175/MWR 3006.1. [Link]

Wentz, F. J., 1993: User's Manual SSM/I Antenna Temperature Tapes Revision 2, RSS Technical Report 120193, Remote Sensing Systems, Santa Rosa, CA.

Ware, R., M. Exner, D. Feng, M. Gorbunov, K. Hardy, B. Herman, Y. Kuo, T. Meehan, W. Melbourne, C. Rocken, W. Schreiner, S. Sokolovskiy, F. Solheim, X. Zou, R. Anthes, S. Businger, and K. Trenberth, 1996: GPS sounding of the atmosphere from low Earth orbit: Preliminary results. Bull. Amer. Meteor. Soc., 77, 19-40, doi: 10.1175/1520-0477 (1996)077<0019:GSOTAF>2.0.CO;2. [Link]

Zou, X., F. Vandenberghe, B. Wang, M. E. Gorbunov, Y. H. Kuo, S. Sokolovskiy, J. C. Chang, J. G. Sela, and R. Anthes, 1999: A ray-tracing operator and its adjoint for the use of GPS/MET refraction angle measurements. J. Geophys. Res., 104, 22301-22318, doi: 10.1029/1999JD 900450. [Link]

Zou, X., B. Wang, H. Liu, R. A. Anthes, T. Matsumura, and Y. J. Zhu, 2000: Use of GPS/MET refraction angles in 3D variational analysis. Quart. J. Roy. Meteor. Soc., 126, 3013-3040, doi: 10.1002/qj.49712657003. [Link] 